\title{
Extracellular vesicles secreted during blastulation show viability of bovine embryos
}

\author{
Edwin A Mellisho, Mario A Briones, Alejandra E Velásquez, Joel Cabezas, Fidel O Castro and \\ Lleretny Rodríguez-Álvarez \\ Laboratory of Animal Biotechnology, Department of Animal Science, Faculty of Veterinary Science, Universidad de \\ Concepcion, Chillan, Chile \\ Correspondence should be addressed to L Rodríguez-Álvarez; Email: Ilrodriguez@udec.cl
}

\begin{abstract}
Extracellular vesicles (EVs) secreted by blastocysts may be clinically relevant, as indicator of embryo viability on in vitro fertilization. We tested if the characteristics of EVs secreted during blastulation are related to embryo viability. Morulae were individually cultured in SOF media depleted of EVs until day 7.5 post IVF. Viable embryos were determined by a system of extended in vitro culture of bovine embryos until day 11 (post-hatching development). Afterward, a retrospective classification of blastocyst and culture media was performed based on blastulation time (early blastulation (EB) or late blastulation (LB)) and post-hatching development at day 11 (viable (V) or non-viable embryo (NV)). A total of 254 blastocysts and their culture media were classified in four groups (V-EB, NV-EB, V-LB, NV-LB). Group V-EB had a larger blastocyst diameter $(\mathbf{1 7 0 . 8} \mu \mathrm{m})$, higher proportion of good-quality blastocysts $(\mathbf{7 7 \%})$ and larger mean size of population of EVs $(122.9 \mathrm{~nm})$, although the highest concentration of EVs $\left(5.75 \times 10^{9} \mathrm{particles} / \mathrm{mL}\right) \mathrm{were}$ in group NV-EB. Furthermore, small RNA sequencing detected two biotypes, miRNA (86-91\%) and snoRNA (9-14\%), with a total of 182 and 32 respectively. In differential expression analysis of miRNAs between V versus NV blastocysts, there were 12 miRNAs upregulated and 15 miRNAs downregulated. Binary logistic regression was used to construct a non-invasive novel model to select viable embryos, based on a combination of variables of blastocyst morphokinetics and EVs characteristics, the ROC-AUC was 0.853. We concluded that characteristics of EVs secreted during blastulation vary depending on embryo quality.

Reproduction (2019) 158 477-492
\end{abstract}

\section{Introduction}

There is increasing evidence of the important roles of secreted extracellular vesicles (EVs) in cell-cell communication; those vesicles can be identified in various biological fluids, including blood and urine, as well as in media from in vitro-cultured cells (van der Pol et al. 2012, Tkach and Thery 2016). Among their biological roles, EVs participate in embryo-maternal crosstalk (Saadeldin et al. 2014, Lopera-Vasquez et al. 2016, 2018, Almiñana et al. 2017, Kurian and Modi, 2019). It has been shown that EVs secreted by the oviduct or the endometrium can modulate the developmental potential of in vitro-produced embryos (Lopera-Vasquez et al. 2017, Lv et al. 2018, Wang et al. 2019). Other reports have identified EVs secreted by embryos, potentially having a role in paracrine communication between cells during early embryonic development (Saadeldin et al. 2014, Mellisho et al. 2017, Qu et al. 2017). However, in the embryo, presence of the zona pellucida (ZP) may modulate secretion of EVs. However, there is scarce information about characteristics of EVs secreted during early embryonic development and how their characteristics may be affected by embryo quality (Ferreira et al., 2013) and developmental stage (Mellisho et al. 2017, Lopera-Vasquez et al. 2018).

Determiningbiological characteristics ofEVs, including variations in their morphology, size, concentration, composition, cellular source and biogenesis, requires use of a combination of technologies (Colombo et al. 2014). EVs contain various types of molecules such as proteins, lipids, mRNA and non-coding RNAs (Braicu et al. 2015). It was recently reported that miRNAs are selectively secreted by the developing human embryos into the culture medium and may be biomarkers of embryonic development (Kropp et al. 2014, Rosenbluth et al. 2014). Also, in this year, Battaglia et al. (2019) demonstrated the presence of miRNA in exosomes from blastocoel fluid. In addition, Rosenbluth et al. (2014) identified miR-191 as highly expressed, in human embryos with chromosomal alterations, with potential as a biomarker for genetic preimplantation diagnosis.

Besides, relationship between embryo competence and developmental kinetics have been reported for early cleavage (Kaith et al. 2015, Milazzotto et al. 2016), early blastulation (Shapiro et al. 2008, Muthukumar et al. 
2013), and early hatching (Utsunomiya et al. 2004). Thus, morpho kinetic parameters may be helpful in improving classical embryo selection (Meseguer et al. 2011, Kaith et al. 2015, Milewski et al. 2016). Early division favors transcript abundance at all stages and increases blastocyst production (Gutiérrez-Adán et al. 2004), whereas early blastulation has been related to better synchrony of embryo development (Hoelker et al. 2006, Shapiro et al. 2008). Blastocyst formation is an essential event in preimplantation embryo development during which many molecular and morphological changes occur (Watson and Barcroft 2001, Madan et al. 2005, Rekik et al. 2011). Between compact morula and blastocyst stages, the first cell lineage differentiation occurs, forming the inner cell mass (ICM) and trophectoderm (Rossant and Tam 2009, Maître 2017).

A critical step in early embryonic development occurs while embryos transit through the oviduct and reach the uterus. During this interval, there is bidirectional communication with the maternal environment to promote development and survival (Zumoffen et al. 2013, Maillo et al. 2016). This communication is mediated by autocrine, paracrine and endocrine signaling (O'Neill 2008). During in vitro culture, effects of co-culture systems on embryonic development reflect existence of embryo tropic factors secreted into the culture medium (Watson et al. 1999, Killian 2004). Several molecules crucial to cellular processes occurring during embryogenesis and embryo communication with its environment have been identified in embryo culture media, including amino acids, fatty acids, metabolites (Seli et al. 2007), proteins (Cortezzi et al. 2011) and miRNAs (Cortezzi et al. 2011, Kropp et al. 2014).

We tested the hypothesis that during blastulation of bovine embryos, morphokinetics and the biological attributes of their secreted EVs (e.g. size, concentration, and small RNA) relating to on embryo viability. Our objectives were to (a) evaluate the differences in blastocyst morphokinetics and the size, concentration and small RNA cargo of EVs secreted during blastulation related to viable and non-viable embryos and (b) establish the potential use of these attributes as a novel predictive model for embryo viability.

\section{Materials and methods}

All experiments were approved by the Ethics Committee of the Faculty of Veterinary Sciences, University of Concepcion (permit no. CBE-17-2017).

\section{Experimental design}

Embryos were cultured in groups ( 25 zygotes per well) until day 5 (D5) of development after in vitro fertilization (morulae stage). At D5, morulae were selected and individually cultured $(80 \mu \mathrm{L})$ in synthetic oviduct fluid media depleted of EVs (SOFdepleted) until D7.5. From D5 to D7.5, embryos were visually monitored for blastocoel formation as an indicator of embryonic development kinetics. This allowed to classify blastocysts as early (day 6.5; experimental group D6.5) or late (day 7.5; experimental group D7.5) blastulating embryos. At day 7.5, morphological characteristics of the blastocysts were determined; embryo quality, developmental stage and blastocyst diameter, using criteria described in the IETS manual (Stringfellow and Givens 2010). Also, at day 7.5, culture media from individual embryos were collected and identified as matched with the corresponding embryo. D7.5 blastocysts were transferred individually to fresh SOFdepleted $(140 \mu \mathrm{L})$ until day 11 (D11) to assess their post hatching developmental (viability) according to growth performance and diameter (Fig. 1).

\section{Experimental groups: according to in vitro post hatching development}

At D11, blastocysts were classified into three categories according to their post hatching: (1) viable (V), size $>270 \mu \mathrm{m}$, quality 1 and 2 ; (2) less viable (LV), size $>160 \mu \mathrm{m}$ and $<270 \mu \mathrm{m}$, quality 3; and (3) non-viable (NV), size $<160 \mu \mathrm{m}$, quality 4 or degenerated. For further binary logistic regression analysis, we consider only the two outermost groups ( $\mathrm{V}$ and $\mathrm{NV}$ ) due to the significant differences existing in their morphological development of the blastocysts at day 11. Embryo culture media from less viable blastocyst were excluded from the analysis to avoid unwanted bias (Fig. 1).

The culture media collected from individual embryos at D7.5 were retroactively assigned to one of the following experimental groups according to the embryo score of D11:

G1: viable with early blastulation (V-EB); $(n=73)$

G2: non-viable with early blastulation (NV-EB); $(n=68)$

G3: viable with late blastulation (V-LB); $(n=61)$

G4: non-viable with late blastulation (NV-LB); $(n=52)$ :

\section{In vitro embryo production}

Ovaries from beef cattle were collected at an abattoir house Frigosur Ltda, Chillan, VIII Region - Bio-Bio located 8 km (15min drive) from the laboratory, following standard procedures described by Rodríguez et al. (2008). The cumulus-oocyte complexes (COCs) were in vitro matured (IVM) in four-well dishes (25-30 COCs per $500 \mu \mathrm{L}$ well) in maturation media for 20-22 $\mathrm{h}$ as described (Velasquez et al. 2016). Oocytes were fertilized using frozen-thawed commercial semen of a beef bull (Semex, Madison, WI, USA) previously used in other in vitro fertilization procedures with good blastocyst production and following standard protocols used in our laboratory (Velasquez et al. 2016). After 18-22 h of IVF, presumptive zygotes were placed in $0.5 \mathrm{~mL}$ of TCM-Hepes $+10 \%$ of fetal bovine serum, cumulus cells were mechanically denuded by 4-min vortexing. Thereafter, they were washed three times before culture.

Presumptive zygotes were in vitro cultured (IVC) in groups in four-well plates $(25-30$ zygotes per $500 \mu \mathrm{L}$ well) in SOF supplemented with $0.37 \mathrm{mM}$ trisodium citrate, $2.77 \mathrm{mM}$ myo-inositol, essential and non-essential amino acids (final concentration $1 \times)$, gentamycin $(50 \mu \mathrm{g} / \mathrm{mL}), 3 \mathrm{mg} / \mathrm{mL}$ essentially 


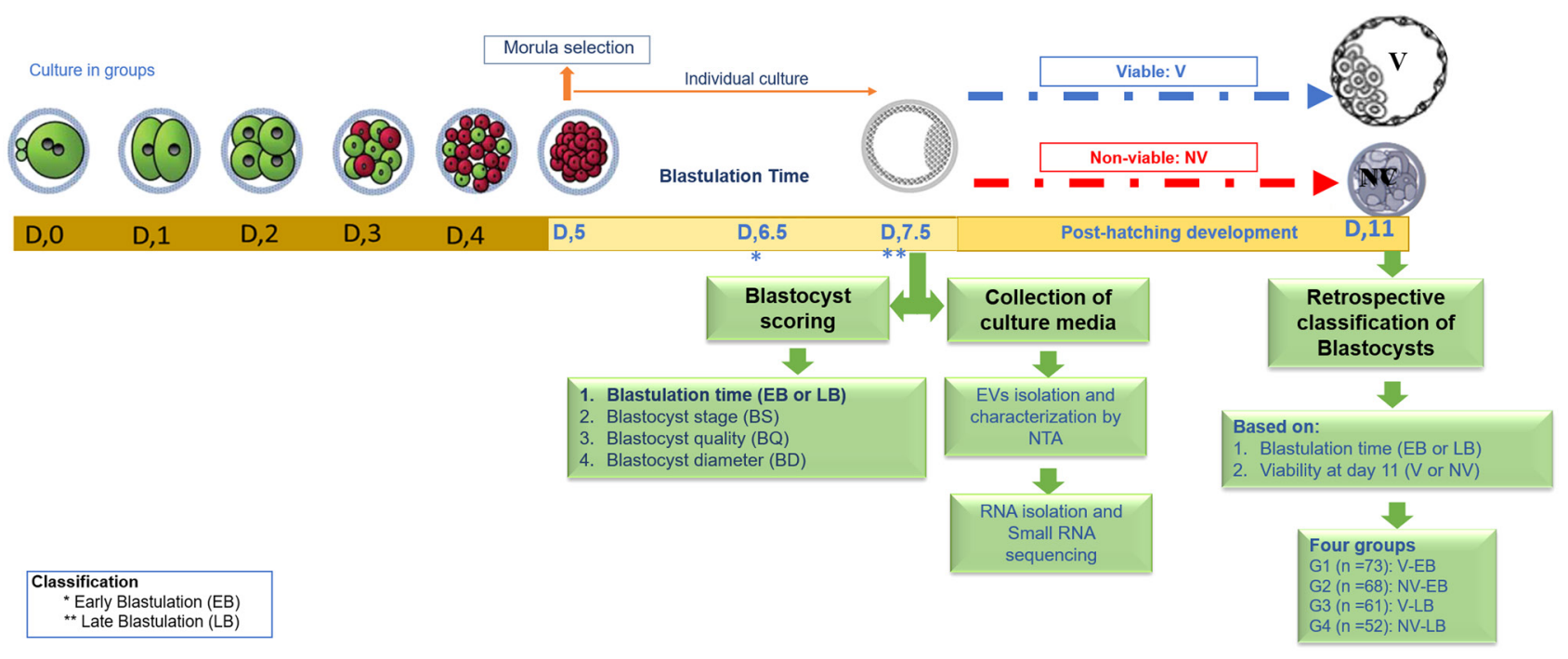

Figure 1 Schematic illustration of experimental design. Morulae (day 5 post IVF) were collected and individually cultured in SOFdepleted up to day 7.5. Embryos at blastocyst stage were determined at day 6.5 (early blastulation) and day 7.5. At day 7.5, all blastocysts were evaluated and the following were determined: blastocyst stage (BS), blastocyst quality (BQ) and blastocyst diameter (BD). All blastocysts were transferred to fresh SOFdepleted and individually cultured up to day 11 . At day 11, blastocysts were classified according to their post-hatching viability (C: viable, NC: non-viable).

fatty acid-free BSA, 2\% fetal bovine serum (FBS) and $10 \mathrm{ng} / \mathrm{mL}$ EGF in a balanced gas atmosphere consisting of $5 \% \mathrm{CO}_{2}, 5 \%$ $\mathrm{O}_{2}$ and $90 \% \mathrm{~N}_{2}, 100 \%$ humidity at $39^{\circ} \mathrm{C}$.

On day 5 post IVF, morulae were selected (stage 3 early morula and stage 4 - morula) using criteria described in IETS manual (Stringfellow and Givens 2010). Morulae were washed three times in SOFdepleted and placed in a 96-well plate $(80 \mu \mathrm{L}$ per well) for individual culture until day 7.5. During this period, embryos were monitored, and the formation of blastocoel was verified (EB at day 6.5 and LB at day 7.5).

On day 7.5, morphology characteristics of the blastocysts were measured; embryo quality and developmental stage and blastocyst diameter, using criteria described in IETS manual (Stringfellow and Givens 2010). Embryo quality was graded based on morphological integrity of embryos (number of cells, degree of fragmentation and regularity, scale from 1 to 4). Codes were defined as follows (Bó and Mapletoft 2013): quality 1 (excellent or good), quality 2 (fair), quality 3 (poor) and quality 4 (dead or degenerating). Embryo diameter was measured using the 5-Mpx Micrometrics digital camera software (Arquimed, Santiago, Chile). On day 7.5, embryo culture media was collected individually and identified with the matching embryo and stored individually at $-80^{\circ} \mathrm{C}$ until analysis. The blastocysts of day 7.5 were transferred to SOFdepleted $(140 \mu \mathrm{L})$ until day 11 to assess their in vitro post hatching developmental viability according to their growth performance and diameter at day 11 (Brandão et al. 2004, Fischer-Brown et al. 2004, Mellisho et al. 2017). On day 11 , morphology characteristics of the blastocysts were measured - embryo quality and developmental stage and blastocyst diameter. Individual embryo culture was carried out at $39^{\circ} \mathrm{C}$ under gas mixture $\left(\mathrm{CO}_{2}, 5 \% ; \mathrm{O}_{2}, 5 \%\right.$ and $\mathrm{N}_{2}, 90 \%$ ).

\section{Isolation of EVs from culture media}

The technique of EVs isolation from individual embryo culture media was previously described by Mellisho et al. (2017). Five hundred microliters of PBS, previously filtered through a $0.22 \mu \mathrm{m}$ syringe filter, were added to culture media collected from individual embryos $(\sim 80 \mu \mathrm{L})$ and centrifuged at $700 \times \mathbf{g}$ for $10 \mathrm{~min}$ (to eliminate cellular debris). The supernatant was subjected to a second centrifugation at $2500 \times \mathbf{g}$ for $20 \mathrm{~min}$ (to eliminate apoptotic bodies). Finally, the recovered supernatant (approximately $500 \mu \mathrm{L}$ ) containing the EVs (microvesicles and exosomes) was analyzed by NTA to determine the concentration and size distribution of nanoparticles in culture media from individual preimplantation embryos.

\section{Nanoparticle tracking analysis (NTA)}

Isolated EVs from individual embryo culture media were analyzed by NTA on a NanoSight NS300 (Malvern Instruments Ltd, UK) equipped with a sCMOS camera. Size distribution and concentration of nanoparticles in liquid suspension was measured. At least 60 particles per frame were measured. Negative controls (PBS and SOFdepleted) had less than seven particles per frame. Sample was injected in continuous sample flow with a syringe pump into the sample chamber at room temperature (RT). Each sample was measured in triplicate with the same camera settings: acquisition time of $60 \mathrm{~s}$ and detection threshold setting of 8 . The detection threshold was similar in all samples. For this analysis, a $488 \mathrm{~nm}$ laser beam was applied to the diluted suspension of vesicles. Data were captured and analyzed using NTA analytical software, version 3.2 Dev Build 3.2.16. Graphical analysis showed particle size distribution of the nanoparticles in the groups and concentration was reported as particles per milliliter. 


\section{Transmission electron microscopy analysis}

Transmission electron microscopy was used to identify EVs. The EVs suspended pellets were deposited on formvarcarbon-coated copper grids for whole-mount preparations and subjected to transmission electron microscope (TEM) as described (Mellisho et al. 2017). Two grids were prepared and washed and fixed in $1 \%$ glutaraldehyde in $0.1 \mathrm{M}$ PBS, and then were contrasted in uranyl-oxalate solution ( $\mathrm{pH} 7.0)$ for $5 \mathrm{~min}$ and methyl cellulose-UA on ice for $10 \mathrm{~min}$. A total of 20 fields and images were processed using ImageJ $1.47 \mathrm{t}$ software. Grids were visualized on a Zeiss EM 900 TEM (Zeiss, Germany) operated at $80-90 \mathrm{kV}$.

\section{Analysis of EV markers}

EVs cannot be used directly in flow cytometry characterization, instead they were bound to latex beads as described (Mellisho et al. 2017). Briefly, EVs $\left(35 \mu \mathrm{L}\right.$ or $4 \times 10^{8}$ particles $\left./ \mathrm{mL}\right)$ were incubated with $4 \mu \mathrm{m}$ aldehyde/sulfate latex beads $(0.125 \mu \mathrm{L}$ or $1.25 \times 10^{5}$ particles $/ \mathrm{mL}$ ) (Life Technologies) in a $100 \mu \mathrm{L}$ final volume of PBS and incubated overnight at $4{ }^{\circ} \mathrm{C}$. Next, $22 \mu \mathrm{L}$ of $1 \mathrm{M}$ glycine/PBS (100 mM final concentration) was added and mixed gently (to block unbound sites of latex beads) and kept for $45 \mathrm{~min}$ at RT. The MVs/beads complexes were washed twice with $1 \mathrm{~mL}$ of PBS/0.5\% bovine serum albumin by centrifugation at $1500 \times \mathrm{g}$ for $3 \mathrm{~min}$ at RT. The MVs/beads complexes were incubated with primary FITC-conjugated antihuman antibodies against CD63 (Abcam, catalog no. 18235, clone MEM-259) or CD9 (Abcam, catalog no. 34162, clone MM2/57) for $1 \mathrm{~h}$ at RT. A negative control antibody reaction was performed using latex beads alone incubated with anti-CD63, anti-CD9, anti-CD81 and anti-CD40L for $1 \mathrm{~h}$ at RT. The labeled $\mathrm{MVs} /$ beads complex were pelleted and washed twice as above with $1 \mathrm{~mL}$ of $\mathrm{PBS} / 0.5 \%$ bovine serum albumin, and finally $100 \mu \mathrm{L}$ pellets were resuspended in $400 \mu \mathrm{L}$ of focusing fluid and subjected to flow cytometry using Attune ${ }^{T M}$ NxT Flow Cytometer (Life Technologies, Inc). EVs isolated from bovine follicular fluid was used as positive control.

\section{Exosome RNA isolation and quantification}

EVs isolated from individual embryo culture media and analyzed by NTA were further processed for small RNA sequencing. For this, three biological replicates and four technical replicates were used yielding 12 samples (G1.1, G1.2, G1.3; G2.1, G2.2, G2.3; G3.1, G3.2, G3.3; G4.1, G4.2, G4.3). Each replicate was the pool of EVs from $\sim 20$ individual embryo culture media. The EVs from each pool were concentrated $(\sim 250 \mu \mathrm{L})$ using Amicon Ultra Centrifugal Filters (Amicon $®$ Ultra-15,100 KDa) to maximize RNA extraction and library preparation. A new filter device (ultracel centrifugal filter device with a $100 \mathrm{~K}$ NMWL $2500 \times$ g) was used for each replica. Immediately, sample pellets were frozen at $-80^{\circ} \mathrm{C}$ pending isolation of RNA. Samples $(n=12)$ were sent on dry ice $\left(-80^{\circ} \mathrm{C}\right)$ for small RNA isolation, quantitation, library preparation, and sequencing using NextSeq 500/550 Mid Output Kit v2 high-throughput sequencing platform (Norgen Biotek Corp, Thorold, ON, Canada). Total RNA was extracted from EVs secreted by bovine blastocysts using plasma/serum RNA Purification Kit (\#55000, Norgen Biotek), the final elution volume was $15 \mu \mathrm{L}$. For RNA quantification, $1 \mu \mathrm{L}$ of RNA was used in a $20 \mu \mathrm{L}$ RiboGreen assay (Norgen's in-house developed modification). miR-21 and 5 S rRNA by RT-qPCR was used as quality control of RNA. One microliter of RNA was used in $5 \mu \mathrm{L}$ TruScript RT reaction using miR-21 stem-loop RT primer or 5S rRNA Reverse Primer. Entire RT reaction was used in $20 \mu \mathrm{L}$ of qPCR reaction using miR-21 or 5S rRNA primers (Supplementary Table 1, see section on supplementary data given at the end of this article).

\section{Small RNA sequencing analysis}

Small RNA isolation, quantitation, quality control, library preparation, sequencing (10M reads per sample) and bioinformatics analysis were outsourced to Norgen Biotek. Small RNA populations were prepared for sequencing using a Multiplex Small RNA Library Prep Set for Illumina following the manufacturer's protocol. The initial step of small RNA-Seq analysis was to evaluate sequence read quality and removal of adapter sequences (Truseq 3': TGGAATTCTCGGGTGCCAAGG). Reference or database sequences used were bostau8 (Genome; https://genome.ucsc. edu/), miRBase version 21 (for miRNAs; release date: 201406-1; https://mirbase.org), Ensembl (for snRNA, snoRNA and rRNA; Bos taurus Genome assembly: UMD3.1; https://www. ensembl.org) and gtRNAdb (for tRNAs; https://gtrnadb.ucsc. edu), RNAdb (piRNAs; https://matticklab.com/rnadb).

sRNA detection (adapter removal, quality control, mapping, counting) was performed using Oasis (V2.0; https://oasis. dzne.de/) (Capece et al. 2015) with the input reads FASTQ files. For differential expression analysis, the module (Oasis) used as input count files that were output from the 'small RNA detection' module. The differential expression analysis powered by DESeq2 (Love et al. 2014) facilitated comparison of miRNAs expression between viable versus non-viable groups.

\section{Prediction of novel miRNA}

Prediction of novel miRNAs was performed with miRDeep2 software (Oasis Platform) in 'sRNA Detection' module reads FASTQ files, annotates known sRNAs, and novel miRNA. Novel predicted miRNAs were saved in Oasis Platform, small RNA database (Dweep et al., 2011).

\section{Target prediction and gene ontology analysis}

The target genes of 11 miRNAs upregulated were predicted using the algorithms in miRWalk database version 3.0 (http:// mirwalk.umm.uni-heidelberg.de/) according to established parameters (Dweep et al. 2011). The novel upregulated miRNA was not included in this analysis. For prediction of miRNA targets by miRWalk, filter set was used to identify the 3'UTR and retained only the cross-database predicted targets (Using miRanda, miRDB, miRWalk and TargetScan database). Embryo viability-associated target genes of the miRNAs upregulated present in EVs formed the basis of miRNA-mRNA interaction network that was visualized using mirWalk graphics available at http://mirwalk.umm.uni-heidelberg.de/graph/. Then, all 
targets were classified according to their function in several ways (gene ontology (GO)): molecular function, biological process and cellular components. Functional annotation of the miRNA target genes was carried out by PANTHER classification system, version 13.1 (Mi et al. 2013). The official gene symbols (example: ENSBTAT00000023657) of miRNA target genes were uploaded in tab-delimited text format to PANTHER available at http://www.pantherdb.org/.

\section{Statistical analysis}

Size, concentration and miRNA cargo of EVs according to embryo viability

Measurable variables of EVs (mean size, mode size and concentration) of each experimental group (G1, G2, G3, G4) were analyzed for normality of distribution by using Kolmogorov-Smirnov test and then compared, with a nonparametric $U$ Mann-Whitney test. Analysis of identified miRNA cargo used data from the filtration cut-off values of five counts per million (CPM5) based on EdgeR algorithm. Data were compared to the 791 mature cattle miRNAs curated in miRBase, version 21 (Bos taurus for miRNAs; release date: 2014-06-1; https://mirbase.org). Selection of differentially expressed miRNAs was based on $\log _{2}$ FC ( $\log _{2}$-fold change) of more than 1.5 (upregulated) or less than -1.5 (downregulated), and with an FDR (false discovery rate) of $\leq 0.05$.

\section{Clustering analysis to discriminate viable of non- viable embryo}

Principal Component Analysis (PCA) was used along with multi-dimensional dataset and cluster analysis (CA) to detect differences among samples of viable and non-viable embryo, applying the same approach previously adopted for dataset (Penkova 2017). In this work, a multivariable dataset of blastocyst morphokinetics (stage of development, quality and diameter of blastocyst at day 7.5), EVs size (mean, mode), concentration and counts of miRNA and snoRNA (CPM>5) were analyzed by PCA and CA among groups of viability and blastulation (G1, G2, G3 and G4). Statistical analysis was performed with SAS system, version 8 for windows (SAS Institute Inc., 1999).

\section{Predictive models of embryo}

The predictive model of embryo viability proposed here was based on binary logistic regression to describe the dichotomousdependent variable of the blastocyst (viable $=1$ and nonviable $=0$ ). A set of independent variables (morphokinetics of blastocysts and characteristic of EVs) were analyzed with multiple regression. Logistic regression generated the coefficients and its standard errors and significance level of an equation to calculate a probability, where values between 0.5 and 1 predict embryo viability. To verify the predictive power of the algorithm, the following indicators were used: analysis of the receiver-operating characteristic (ROC) with determination of area under the curve (AUC), percentage correct predictions, and Omnibus tests. Statistical significance was determined at the $P<0.05$ level. A rough guide for classifying the accuracy of a predictive model is $0.90-1=$ excellent, $0.80-0.90=$ good, $0.70-0.80=$ fair, $0.60-0.70=$ poor, $0.50-0.60=$ fail. Statistical analysis was performed with the program IBM SPSS Statistics, version 19 (IBM).

\section{Results}

\section{Blastocyst morphokinetics according to embryo viability}

A total 4665 COCs of quality 1 and 2 were used for in vitro fertilization, producing 1371 good-quality morulae in group culture (efficiency 29.4\%). Morulae were further cultured individually in EVs-depleted media, up to the blastocyst stage. There were 230 blastocysts at day 6.5 (early blastulation, $16.8 \%$ ) and 327 blastocysts at day 7.5 (LB; $23.9 \%$ ). In all, $40.6 \%$ of morulae developed to blastocyst. At day 7.5, all blastocysts independent of the timing of reaching that state were collected and kept in extended individual culture up to day 11. At day 11, 254 embryos were selected and based on their developmental performance were divided into four groups: $\mathrm{V}-\mathrm{EB}, \mathrm{NV}-\mathrm{EB}$, V-LB, NV-LB. Non-hatched blastocysts were discarded. Diameter at day 7.5 was larger in V-EB $(170.8 \mu \mathrm{m}$; $\mathrm{p}<0.05$ ) (Fig. 2; Supplementary Table 2). No differences in diameter were observed in day 7.5 blastocysts from groups NV-EB and V-LB; however, blastocysts from NV-LB were the smallest embryos at that stage (146.5 $\mu \mathrm{m}$; $P<0.05$ ) (Fig. 2; Supplementary Table 2). In addition,

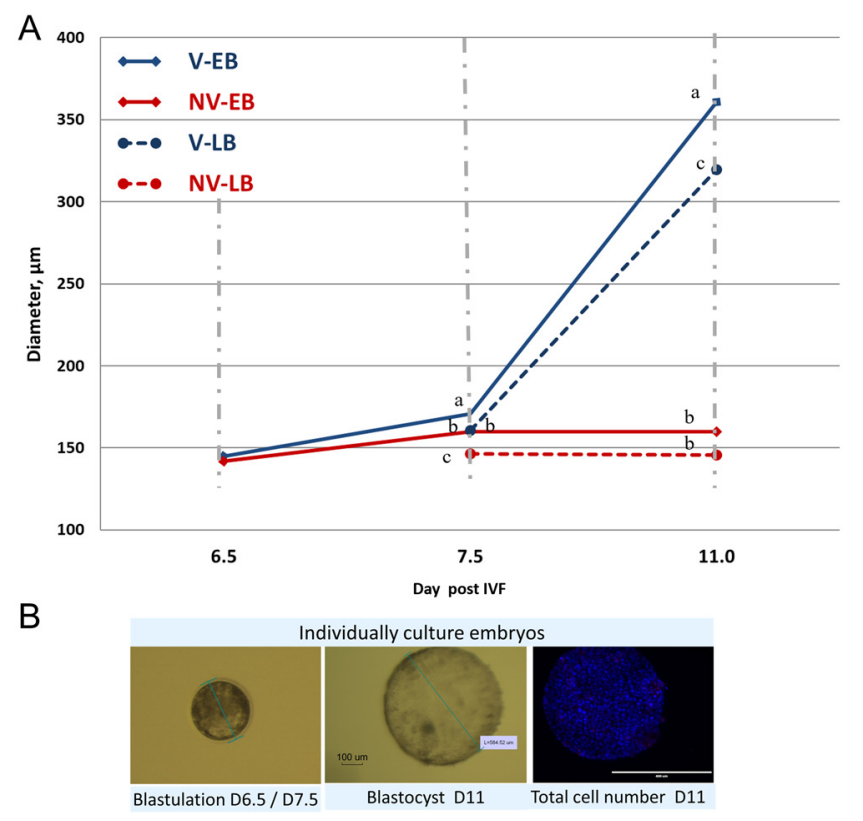

Figure 2 Blastocysts growth from day 6.5 up to day 11. (A) Viable embryos despite their time of blastulation showed a lineal growth from day 7.5 up to 11 , compared to non-viable embryos. Different letters within the same developmental day $(6.5,7.5$ or 11$)$ indicate statistical differences $(P<0.05)$. (B) Representative pictures from blastocyst morphology (days 6.5 and 7.5) during blastulation time and post-hatching development (day 11). 
V-EB group was the only one with hatched blastocysts at day 7.5, and it had the highest percentage of best quality blastocysts at day 7.5 (77\% quality 1 blastocysts and $10 \%$ in hatching stage (Supplementary Table 2)). Conversely, embryos from NV-LB group showed delayed development; $96.1 \%$ of the blastocysts at day 7.5 were at early stage and $37 \%$ of embryos were classified as quality levels 3 and 4 (Supplementary Table 2).

\section{Size, concentration and small RNA cargo of EVs according to embryo viability}

The NTA analysis characterized populations of EVs in terms of size and concentration. The EVs analyzed were secreted during the transition from morulae to blastocyst (day 5-7.5; Fig. 3A). Filtered PBS solution and SOF-depleted media used for embryo culture were included as negative control: these solutions had very low concentration of particles that did not influence characterization of EVs populations secreted by individual embryos (Fig. 3B). Isolated EVs were visualized by TEM (Fig. 3C) and confirmed as EVs by immunoidentification of surface markers CD9, CD81, CD63 and CD40L (Fig. 3D).

Population of EVs showed significant differences among groups of embryos, with the highest mean size corresponding to EVs from V-EB embryos $(122.90 \mathrm{~nm}$; $P<0.05)$ (Fig. $3 \mathrm{~A}$ and B; Supplementary Table 3). However, there were not significant differences between viable and non-viable embryos with LB. Regarding
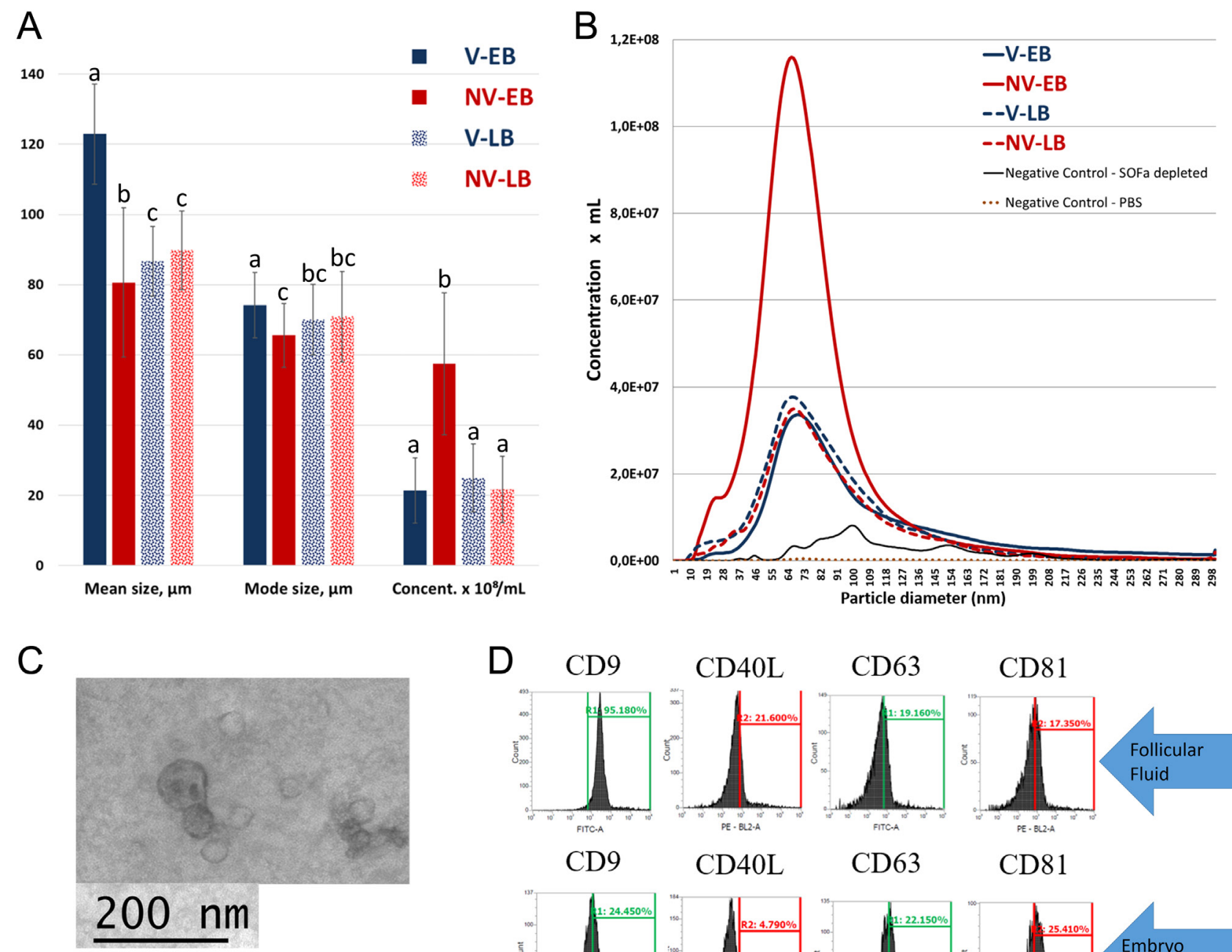

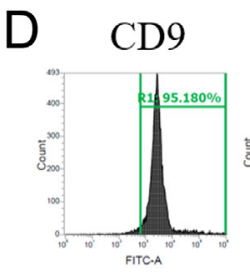

CD9

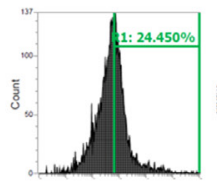

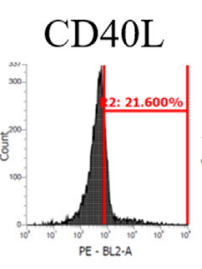

CD40L

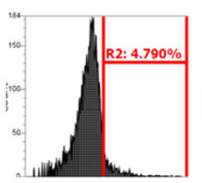

CD63

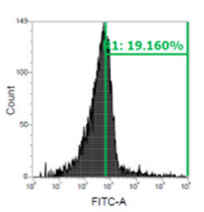

CD63

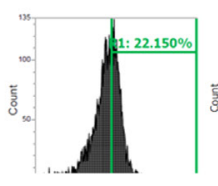

CD81

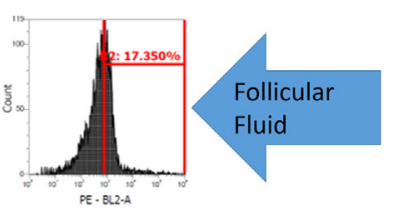

CD81

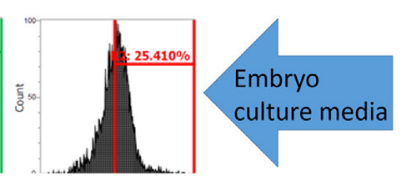

Figure 3 Measurements of the size and concentration of EVs secreted from bovine blastocyst. (A) Size and concentration of EVs populations measured with Nanoparticle tracking analysis (NS300). In A, Y-axis indicates concentration ( $10^{8}$ particles $\times$ ml), while X-axis indicates size distribution $(\mathrm{nm})$. Depleted SOF media and filtered PBS were included as negative controls. (B) Descriptive statistical values (mean, mode, concentration) of EVs populations and comparison measures between viable versus non-viable embryo groups. Different letters within EVs characteristic mean statistical differences with $P<0.05$. (C) Representative pictures from transmission electron micrographs showing morphology of EVs secreted by bovine blastocysts and (D) flow cytometry analysis of EVs markers (CD9, CD63, CD81, CD40L). 
EVs concentration, culture media from NV-EB had the highest concentration of EVs $\left(5.75 \times 10^{9}\right.$ particles $\left./ \mathrm{mL}\right)$ (Fig. 3A and Supplementary Table 3).

High-throughput sequencing was used to determine small RNA content in $\mathrm{EV}$ s derived from preimplantation bovine embryos during blastulation. Overall 14.9 million raw reads (ranging from 9 to 18 million) were achieved. After bioinformatics processing, $0.24-3.03 \%$ were mapped as unique reads. The most abundant biotypes of small RNA were miRNA (86-91\%) and snoRNA (9-14\%) (Fig. 4A). Bioinformatics analysis identified a total of 182 miRNAs; 161 were known miRNAs and 21 putative novel miRNAs (p-bta-miR-) (Table 1). For this identification, the cut-off was set at five counts in EVs from embryo culture media. These newly identified miRNAs have apparently not been reported in other species. Further, miRNAs/ snoRNAs counts were compared between viable and non-viable embryos. Eight miRNAs (bta-miR-1, bta-miR-
A

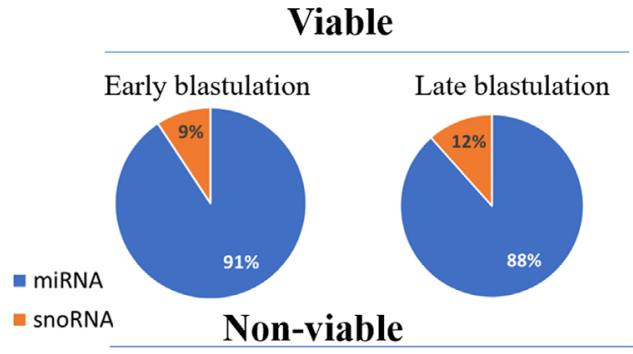

Early blastulation

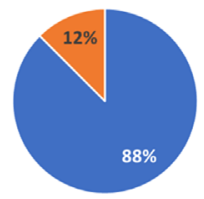

Late blastulation

C

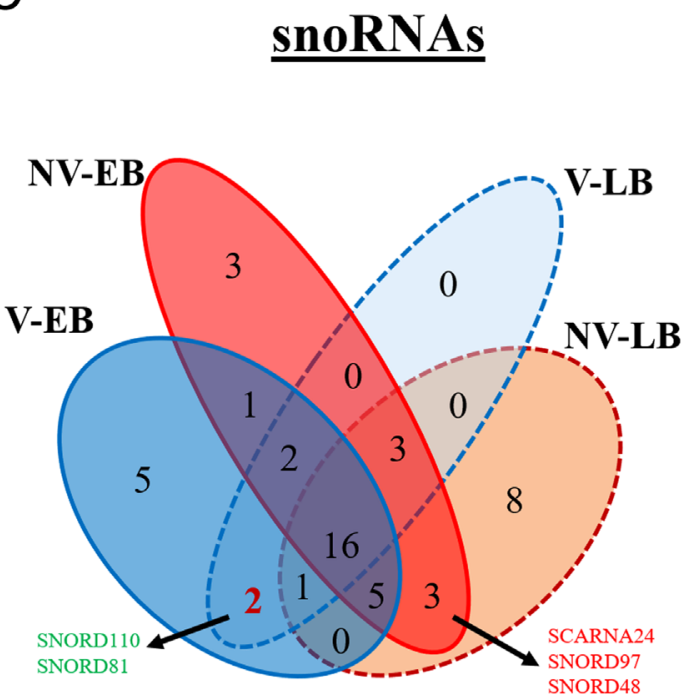

B

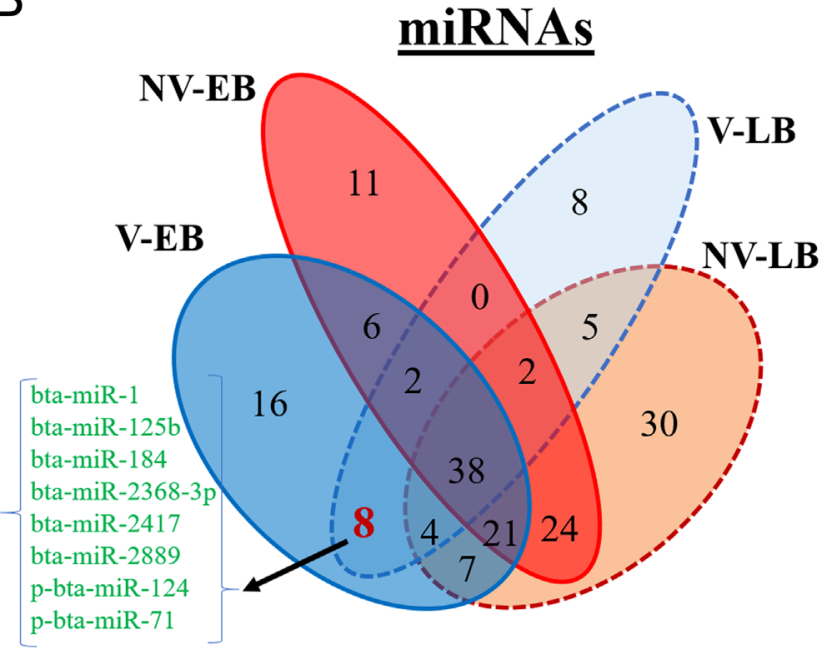

D

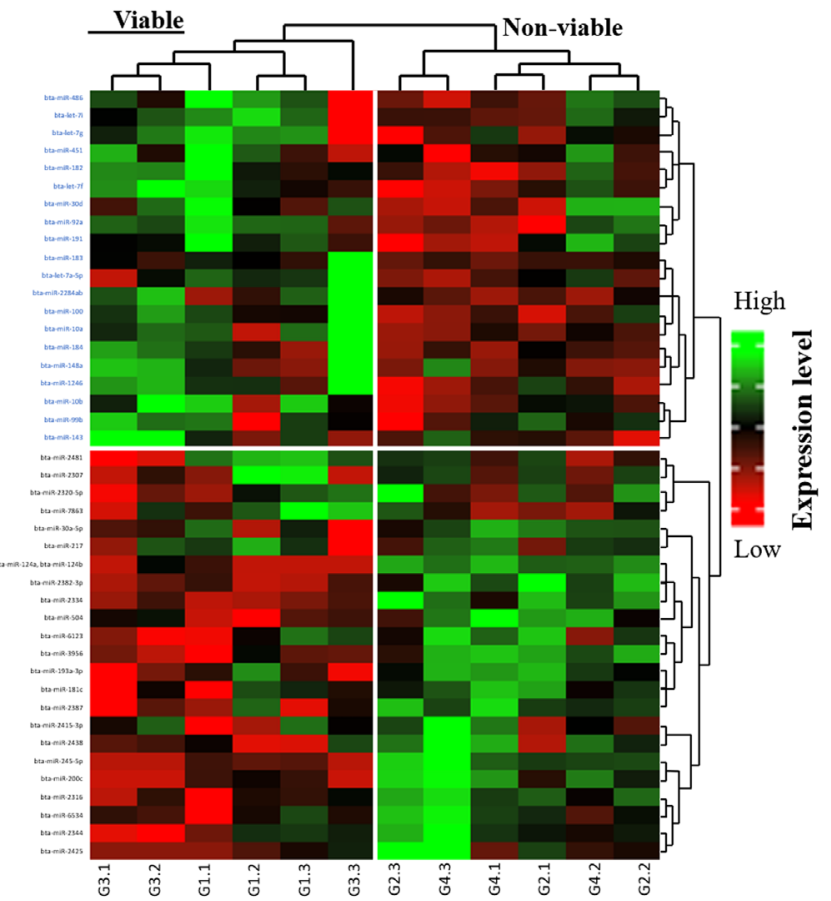

Figure 4 Distribution of small RNA species in EVs secreted by preimplantation bovine embryo during blastulation (from day 5 up to day 7.5 of embryo development). (A) Distribution of small non-coding RNAs obtained from EVs from blastocyst according to their viability. (B and C) Venn diagram depicting counts of miRNAs and snoRNAs (CPM>5) found in EVs from viable (blue/green circle) and non-viable embryos (red/black circle). (D) Heatmap profile of miRNA counts (CPM>5) from EVs secreted by bovine blastocysts. Global color scale shows upregulated (green) and downregulated (red) miRNA expression levels. 
Table 1 Identification of a novel miRNAs (CPM $\geq 5$ per sample) of EVs from embryo culture media.

\begin{tabular}{|c|c|c|c|c|c|c|}
\hline Mature candidate ID* & Mature sequence & Length & Chromosome & Mature start & Mature end & Mature strand \\
\hline p-bta-miR-101 & AGGGUCAGGUAAGGGUCUCCACU & 23 & chr5 & 55981753 & 55981775 & + \\
\hline p-bta-miR-110 & UUGUCCCUGGUCGCGGCCUCUGC & 23 & chr7 & 5779012 & 5779034 & + \\
\hline p-bta-miR-119 & GGUUAGCCAGGACACAGCGGAG & 22 & chr4 & 115606058 & 115606079 & - \\
\hline p-bta-miR-124 & UUUCACCCGGUCUCUUUGGCUC & 22 & chr4 & 5633784 & 5633805 & + \\
\hline p-bta-miR-129 & CCGAGCGCGGACCUCCGGCGCCU & 23 & chr24 & 50939210 & 50939232 & + \\
\hline p-bta-miR-134 & UGUGGUCUGUGUUCUGUAAGA & 21 & chr1 & 75157296 & 75157316 & + \\
\hline p-bta-miR-14 & UGGCACCAGCACUGGCGGUGGC & 22 & chr26 & 37549333 & 37549354 & - \\
\hline p-bta-miR-20 & GCACGGGUGGUUCAGUGGUA & 20 & chr2 & 41564663 & 41564682 & + \\
\hline p-bta-miR-24 & UCCAGGAUAUGUGCGUGUAACU & 22 & chr9 & 24769364 & 24769385 & - \\
\hline p-bta-miR-26 & UGCACCACCCGUGGUCACUCCU & 22 & chr23 & 28502263 & 28502284 & - \\
\hline p-bta-miR-28 & AUCGCGACGGGUAUCUCU & 18 & chr11 & 11314991 & 11315008 & - \\
\hline p-bta-miR-31 & AAAACCUCAACGAACUCUUUGG & 22 & chr27 & 15045875 & 15045896 & + \\
\hline p-bta-miR-34 & UUAGGAUUGACAGAUUGA & 18 & chr10 & 34366159 & 34366176 & + \\
\hline p-bta-miR-35 & CCGGGCCUGGGCCGAGCCCCA & 21 & chr15 & 36372562 & 36372582 & - \\
\hline p-bta-miR-39 & UCGAGUGCUGAAGUGACCUUCU & 22 & chr21 & 7226582 & 7226603 & + \\
\hline p-bta-miR-47 & GGACAGUGUCAGUUGGGCA & 19 & $\operatorname{chrX}$ & 113743090 & 113743108 & - \\
\hline p-bta-miR-58 & UAAGACCUCACAUGCCUUGCAGC & 23 & chr19 & 41310531 & 41310553 & + \\
\hline p-bta-miR-61 & CGCGCUGCAUGCCGGGAGGCU & 21 & chr13 & 63816904 & 63816924 & - \\
\hline p-bta-miR-69 & UCCUCAGUAGCUCAGUGGUAAA & 22 & chr12 & 82691418 & 82691439 & + \\
\hline p-bta-miR-71 & UAGAGCAGACGACUCAGAUACCG & 23 & chr20 & 5593899 & 5593921 & + \\
\hline p-bta-miR-72 & UUCGGGCGGCGCGGGUCCCGGG & 22 & chr10 & 9674464 & 9674485 & - \\
\hline p-bta-miR-85 & CGCCCUUCUGUACUCGCGCCCG & 22 & chr11 & 107268646 & 107268667 & - \\
\hline
\end{tabular}

*Nomenclature oasis, predicted.

125b, bta-miR-184, bta-miR-2368-3p, bta-miR-2417, bta-miR-2889, p-bta-miR-124, p-bta-miR-71) were exclusively detected in media from viable embryos (including EB and LB) (Fig. 4B). Conversely, there were 24 exclusive miRNAs in media from non-viable embryos (Fig. 4B).

A total of 32 snoRNAs identified (Fig. 4C), of which two (SNORD110, SNORD81) were exclusively present in media from viable embryos (including early and LB), whereas media from non-viable embryos had three exclusive snoRNAs (SCARNA24, SNORD97, SNORD48). Heatmap (Fig. 4D) illustrated that viable and non-viable embryos had a differential miRNA expression pattern $(F D R<0.05)$.

For differential expression analysis, data were filtered by $\log _{2}$ ratio $>1.35$ or $<-1.35, P$ value adjusted (FDR $)<0.05$ and $P<0.05$. Based on expression level analysis of each miRNA, 27 of the 182 identified miRNAs were confirmed to exhibit significantly differential expression levels in viable versus non-viable embryos, despite blastulation time (Fig. 5 and Supplementary Table 4). Among these miRNAs, 12 miRNAs had higher expression levels in viable embryos (Fig. 5A), whereas the remaining 15 miRNAs were downregulated in these embryos (Fig. 5B). The miRNAs deregulated the most, according to the $\log _{2}$ fold change $(\mathrm{FC})$ of expression were bta-miR-2284ab ( $\mathrm{FC}=4.6)$, bta-miR-124a and bta-miR$124 \mathrm{~b}(\mathrm{FC}=-3.5)$ (Supplementary Table 4). Five of the 12 miRNAs with a higher expression level were exclusively identified in viable embryos (bta-miR-1, bta-miR-184, bta-miR-2368-3p, bta-miR-2889, p-bta-miR-71). When miRNAs from embryos that reached blastulation at different time (early or late) were compared considering the same criteria (FDR $<0.05$ and $P<0.05)$, no differentially expressed miRNAs were found despite embryo viability. Among the 32 snoRNAs identified, one (SNORD20 ENSBTAG00000043297) was upregulated $(\mathrm{FDR}<0.05)$ in EVs of viable embryos compared to nonviable embryos (Supplementary Table 5).

Potential target genes of 11 known miRNA upregulated (Fig. 5C) and 12 known miRNAs downregulated (Fig. 5D) in viable embryos were identified using miRWalk 3.0 database, yielding 9890 and 14307 target genes, respectively, with miRNA potential binding sites in their $3^{\prime}$ UTR respectively. Putative novel miRNAs $(n=4$; p-btamiR-14, p-bta-miR-26, p-bta-miR-61, p-bta-miR-71) were not included in this analysis, as no target genes are known at present. In upregulated, three miRNAs can potentially regulate $58 \%$ of target genes: btamiR-2449 (2477 genes), bta-miR-320a (1947 genes) and bta-miR-7863 (1246 genes) (Fig. 5C). Therefore, the interaction network between miRNA and target genes is common in cellular mechanisms (example: predicted target genes and upregulated miRNAs were used to construct the interaction network using the MirWalk graphic software (Fig. 5E)). All targets were classified according to their function in two categories of GO: molecular function (Fig. 5F) and biological process (Fig. 5G) using PANTHER classification system. In $\mathrm{GO}$ annotation for molecular function, most target genes $(84 \%)$ were related to binding (GO:0005488), catalytic activity (GO:0003824) and transporter activity (GO:0005215). In GO annotation for biological process, most target genes (69\%) were related to binding cellular process (GO:0009987), metabolic process (GO:0008152), biological regulation (GO:0065007) 
A

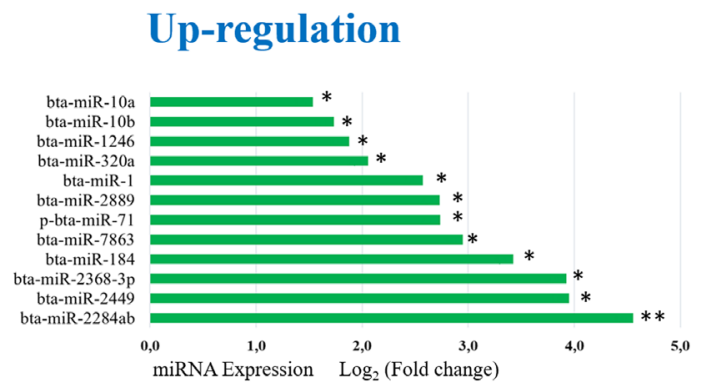

\section{C}

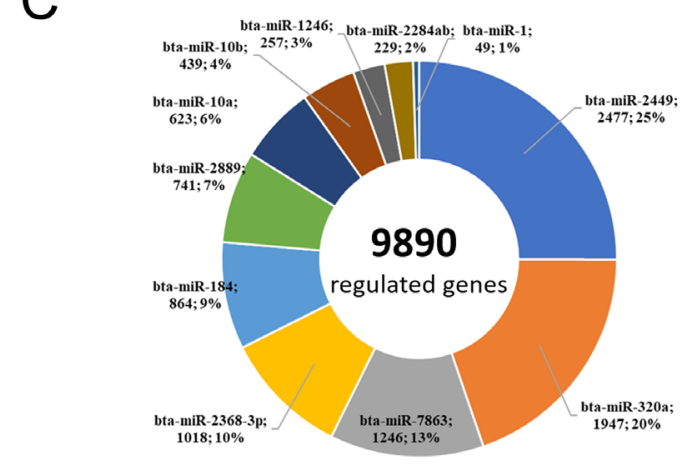

$\mathrm{E}$

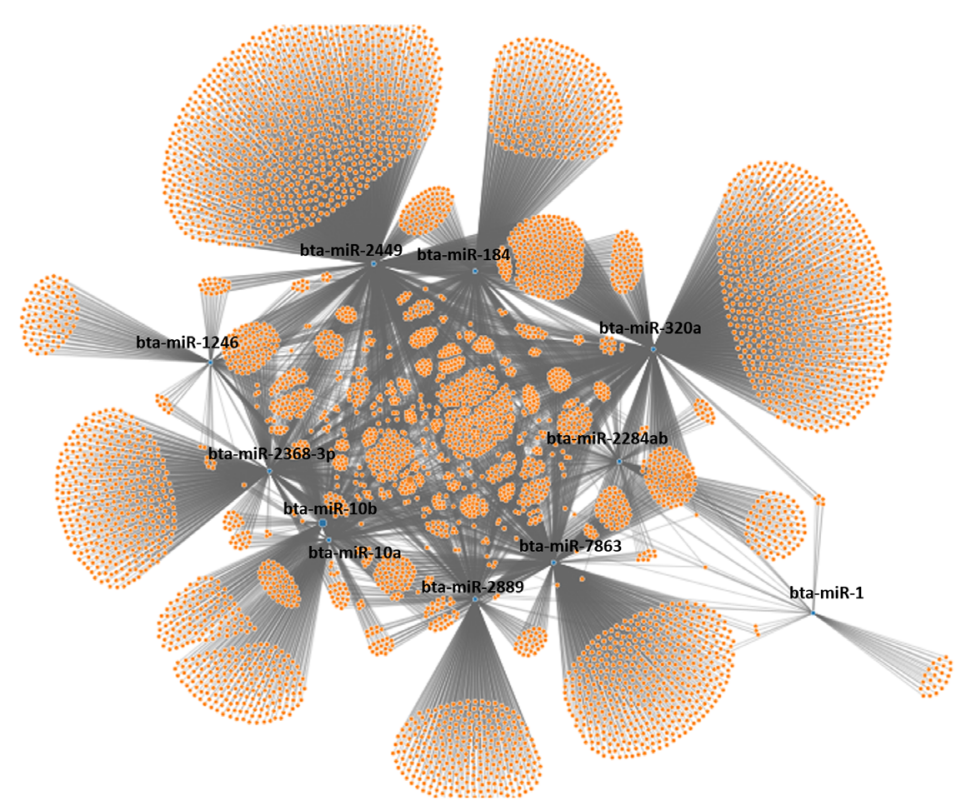

B

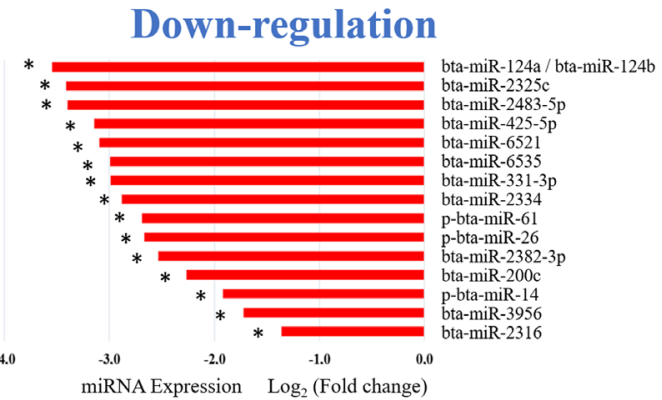

Figure 5 Differential miRNA expression profiles in EVs and the target gene analysis. From 27 miRNAs, 12 are upregulated in viable embryos (A) and 15 are downregulated in these embryos (B). ${ }^{* *}$ indicated statistical significance with corrected FDR $<0.01 ; *$ indicated statistical significance with corrected FDR $<0.05$. (C and D) Prediction of potential target genes for 11 known miRNAs upregulated (C) and 12 known miRNAs downregulated (D) using MirWalk 3.0 database. (E) Interaction network among target genes of 11 miRNAs upregulated (bta-miR2284ab, bta-miR-2449, bta-miR-2368-3p, bta-miR-184, bta-miR-7863, bta-miR-2889, bta-miR-1, bta-miR-320a, bta-miR-1246, bta-miR-10b, bta-miR-10a): Orange nodes indicate a target gene; Blue nodes indicate a miRNA with upregulated expression. (F) Nine significantly enriched GO for molecular function were achieved using the target genes of upregulated miRNAs and (G) twelve significantly enriched GO for biological process were achieved using the target genes of upregulated miRNAs. 

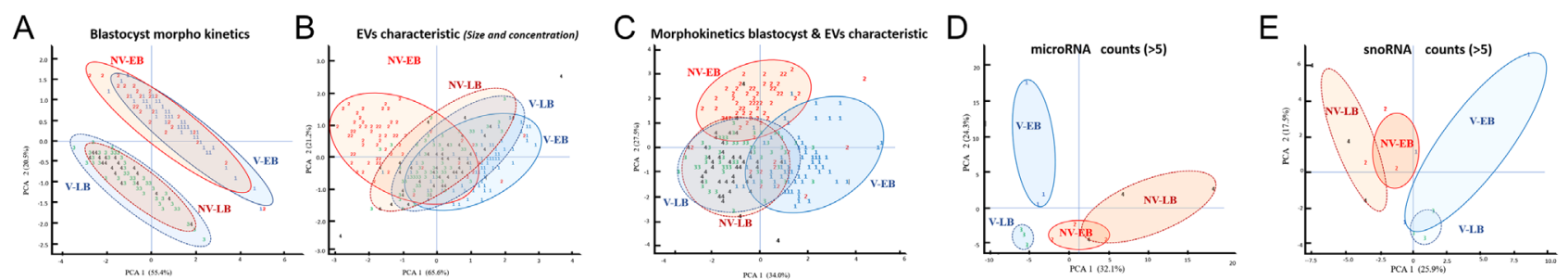

Figure 6 Clustering analysis of blastocyst morphokinetics and EVs properties identifies viable preimplantation embryos. PCA plot and clustering analysis for four groups according to developmental viability and blastulation time (V-EB, NV-EB V-LB and NV-LB): (A) clustering according to blastocysts morphokinetics data, (B) clustering according to EVs characteristic (size and concentration), (C) clustering combining variables from blastocyst morphokinetics and EVs characteristic (size and concentration), (D) clustering according to counts of miRNA and (E) snoRNA (RNA) cargo of EVS from embryo culture media. EB, early blastulation; LB, late blastulation; NV, non-viable; V, viable.

and response to stimulus (GO:0050896). Gene ontology analysis for target genes of downregulated miRNAs had similar results (data not shown).

\section{Clustering analysis of blastocyst morphokinetics and EVs characteristic}

A multivariate data analysis using PCA and CA was performed including different variables (Fig. 6). Blastocysts morphokinetics data (stage, quality and diameter of blastocyst at day 7.5) allowed identification of two groups, discriminating according to moment of blastulation (EB vs LB), but not according to embryo viability (V vs NV) (Fig. 6A). EVs characteristics (mean size, mode size and concentration) allowed to discriminate between blastocyst from V-EB and NV-EB but not between embryos at LB (Fig. 6B). However, when blastocyst morphokinetics data and EVs characteristic are combined in the same analysis, there is a better discrimination of viable and non-viable embryos from early blastulation group (Fig. 6C). Counts of miRNA and snoRNA $(C P M>5)$ were performed in three replicates per group, with a marked discrimination among the four groups for both embryo viability and blastulation time (V-EB, NV-EB, V-LB and NV-LB) (Fig. 6D and E).

\section{Non-invasive model to predict embryo viability}

Binary logistic regression was used to construct noninvasive models to predict embryo viability, based on the values of seven variables, including blastocyst morphokinetics characteristics (blastulation time (BT), stage (BS), quality (BQ) and diameter (BD) of blastocyst at day 7.5) and EVs characteristic (mean size (ME), mode of size (MO) and particle concentration (CO)), measured in 254 embryos. In Model-1, all independent variables were analyzed; the most significant predictors associated with embryo viability identified for this model were blastocyst stage, blastocyst quality, blastocyst diameter, mean size of EVs and concentration of EVs $(P<0.05)$ (Table 2). Mode size of EVs and BT were not significant in Model-1.

Model-2 included most significant variables from Model-1 (Supplementary Tables 6 and 7), whereas
Model-3 included highly significant variables $(P<0.011)$. In Model-4 and 5, EVs characteristic were excluded; Model-4 considered all variables from the embryo, whereas Model-5 excluded BT (Table 2). Based on statistical test of predictive power, Model-1 and 2 achieved a ROC-AUC of $0.853(95 \% \mathrm{Cl}, 0.806-0.900$; $P<0.001)$ and correct predictions of 77.2 and $77.6 \%$ respectively (Table 2, Supplementary Table 6 and Supplementary Fig. 1). Model-3 with only three variables had an ROC-AUC similar to Model-1 and 2, but a lower percent of correct prediction (Table 2). Conversely, the analysis of predictive power for Models-4 and 5, achieved an ROC-AUC of $0.714(95 \% \mathrm{Cl}, 0.651-0.777$; $P<0.001)$ and $0.724(95 \% \mathrm{Cl}, 0.662-0.786 ; P<0.001)$ and correct predictions of 65.4 and $66.9 \%$ respectively, indicating the value of EVs characteristics to predict embryo viability (Table 2 and Supplementary Fig. 1).

\section{The logistic function (logit) for Model-2:}

$$
\begin{aligned}
\mathrm{Y}=\ln [p /(1-p)]= & -2.280+(-0.868)^{*} \mathrm{BS} \\
& +(-0.470) * \mathrm{BQ}+(0.047) * \mathrm{BD} \\
& +(0.027) * \mathrm{ME}+(-0.051) * \mathrm{CO}
\end{aligned}
$$

The estimated probability is:

$$
p=\operatorname{Exp} Y /(1+\operatorname{Exp} Y)
$$

Where:

In is the natural logarithm, log exp, where $\exp =2.71828 \ldots$

$\ln [p /(1-p)]$ is the log odds ratio, or 'logit'

$p /(1-p)$ is the 'odds ratio'

$p$ is the probability that the event $\mathrm{Y}$ occurs, $p(\mathrm{Y}=1)$

If $p>0.5$, predict embryo viability

If $p<0.5$, predict embryo non-viability

\section{Discussion}

Recently we and others reported secretion of EVs by bovine, porcine and human embryos (Ferreira et al. 2013, Saadeldin et al. 2014, Mellisho et al. 2017, Battaglia et al. 2019). EVs are recognized as carriers 
Table 2 Predictive non-invasive models of embryo viability with the use of binary logistic regression.

\begin{tabular}{|c|c|c|c|c|c|}
\hline Variables & $\begin{array}{l}\text { Model-1 (full } \\
\text { variables) }\end{array}$ & $\begin{array}{l}\text { Model-2 (significant } \\
\text { variables) }\end{array}$ & $\begin{array}{l}\text { Model-3 (min. } \\
\text { significant variables) }\end{array}$ & $\begin{array}{l}\text { Model-4 (blastocyst } \\
\text { morphokinetics) }\end{array}$ & $\begin{array}{l}\text { Model-5 (blastocyst } \\
\text { morphology) }\end{array}$ \\
\hline Constant & -1.662 & -2.280 & -6.386 & -0.819 & -0.585 \\
\hline \multicolumn{6}{|l|}{ Blastocyst kinetics } \\
\hline Blastulation time $($ early $=1$, late $=0)$ & -0.622 & - & - & -0.472 & - \\
\hline \multicolumn{6}{|l|}{ Blastocyst morphology } \\
\hline Day 7.5; blastocyst stage & -0.874 & -0.868 & - & -0.672 & -0.760 \\
\hline Day 7.5; blastocyst quality & -0.509 & -0.470 & - & -0.530 & -0.477 \\
\hline Day 7.5; blastocyst diameter & 0.048 & 0.047 & 0.036 & 0.039 & 0.039 \\
\hline \multicolumn{6}{|l|}{ EVs characteristics } \\
\hline Mean of EVs & 0.046 & 0.027 & 0.025 & - & - \\
\hline Mode of EVs & -0.034 & - & - & - & - \\
\hline Concentration of EVs & -0.041 & -0.051 & -0.053 & - & - \\
\hline \multicolumn{6}{|l|}{ Power of predictive algorithm } \\
\hline Accuracy of model (ROC-AUC) & 0.853 & 0.853 & 0.834 & 0.724 & 0.714 \\
\hline \multicolumn{6}{|l|}{ AUC: $0.80-0.90$, model good } \\
\hline $\begin{array}{l}\text { Percent correct predictions (bigger, } \\
\text { better the model) }\end{array}$ & $77.2 \%$ & $77.6 \%$ & $72.4 \%$ & $65.4 \%$ & $66.9 \%$ \\
\hline $\begin{array}{l}\text { Omnibus tests of coefficients and } \\
\text { model } P<0.01\end{array}$ & 0.001 & 0.001 & 0.001 & 0.001 & 0.001 \\
\hline
\end{tabular}

Note: Values in the columns corresponded to coefficients of the variable in the logistic function of each model. Below the logistic function of Model-2 is represented. This model had significant variables and the highest percentage of correct predictions.

of proteins, lipids, and nucleic acids and can transfer this cargo to recipient cells, participating in cell-to-cell communication (Raposo and Stoorvogel 2013, Kowal et al. 2014, Battaglia et al., 2019). Although EVs have not been as intensively studied during preimplantation embryo development as in other cell types, it seems that EVs characteristics (mean size, mode of size and particle concentration, as well as their cargo) can be associated with embryo stage and quality embryos (Ferreira et al. 2013, Saadeldin et al. 2014, Mellisho et al. 2017). In fact, we reported that parthenogenetic and IVF embryos secreted dissimilar populations of EVs (Mellisho et al. 2017). In the same research, we also observed a high variability in characteristics of EVs secreted by nonviable blastocyst, whereas viable embryos had less polydispersity of EVs (Mellisho et al. 2017). Skog et al. (2008) and Sharma et al. (2011) mentioned that polydispersity can be indicative of the origin of the vesicles; for example, tumor-derived $\mathrm{EV}$ s had greater polydispersity than those from healthy cell lines.

Based on that information, in this work, we investigated the potential of characterization of $\mathrm{EVs}$ as a non-invasive marker of bovine embryo viability. Using the described experimental design and the adjustment of protocols to isolate and characterize EVs, it was possible to derive information from individual embryos. EVs population from viable and non-viable embryos showed high variability in diameter (range 10-300 nm) and concentration, what is not observed in EVs-depleted media used as negative control for NTA analyses. This diameter range is in concordance to previous report for bovine (Mellisho et al. 2017), human (Ferreira et al. 2013) and porcine (Saadedin et al. 2014) embryos. However, PCA and cluster analysis allowed discriminated viable embryos with early blastulation group, but not at LB.
Perhaps this was due to a difference in EVs secretion depending on embryo developmental kinetic.

In bovine embryos, there is a peri-implantation period when growth and many molecular and morphological changes occur despite a lack of direct contact with the maternal endometria (Graf et al. 2014). Therefore, bovine embryos maintained in a culture system after blastocyst stage to evaluate post hatching development are a convenient model to study embryo viability (Brandão et al. 2004, Velasquez et al. 2016, 2017). To accomplish this, conventional embryo culture system was modified; first embryos were cultured in groups until morula stage to promote development, whereas thereafter, morulae were cultured individually in EVsdepleted media. This facilitated the assessment of development performance of individual embryos and avoid contamination of EVs from FBS (Kropp et al. 2014, Kropp and Khatib 2015, Mellisho et al. 2017). However, under this experimental design, embryos were exposed to non-optimal culture conditions, consequently, the blastocyst rate was reduced from above $30 \%$ in routine laboratory group culture (unpublished) to $\sim 12 \%$. Most embryos blastulated early at day 6.5 , this favored greater blastocyst diameter at day 7.5 (average $=170.8 \mu \mathrm{m}$ ) and post-hatching development. Also $32 \%$ of early blastulated embryos were later classified as viable (V-EB), whereas $16 \%$ of late blastulated embryos were viable (V-LB).

Prospective use of morphokinetics to improve selection by using early (Wong et al. 2010, Motato et al. 2016) or late development parameters have been reported (Campbell et al. 2013). In the present study, both morphokinetic and morphology variables of blastocyst at day 7.5 were significantly related to embryo viability. The prediction power of both models 
(Model-4 and Model-5) achieved an ROC-AUC of 0.714 and 0.72 , respectively, being statistically considered as 'fair' models to select viable embryo. Similar results were reported using models based on time-lapse embryo monitoring, achieving an ROC-AUC of 0.7 (Swain 2013), 0.602 (Motato et al. 2016), 0.70 (Milewski et al. 2015) and 0.71 (Milewski et al. 2016), whereas a model that considers blastocyst morphology, achieved an ROC-AUC of 0.56 for implantation prediction (Motato et al. 2016).

Limitations of embryo viable prediction based on embryo morphology and/or morphokinetics led researchers to pursue more accurate approaches. During early development, embryos interact with the environment. In vitro, embryos consume substrates and oxygen from the culture system while secreting proteins and other molecules, thus changing characteristics of the culture media (Ménézo et al. 2013). The secretome of the oocyte/embryo likely reflects their biochemical or epigenetic status (Katz-Jaffe and Gardner 2008). This information has been used to determine an association between embryo metabolomics or secretion and their developmental competence, in order to design noninvasive models for embryo scoring. For instance, in human embryos, Seli et al. (2011) and Dominguez et al. (2015) proposed a strategy combining timelapse and proteome or metabolomic analysis in spent culture media. In this species, extreme values for ROC analysis were published when comparing metabolomic (NIR spectroscopy, AUC $=0.92$ ) versus morphology score $(A \cup C=0.55)$ for pregnancy prediction ( $\mathrm{Li}$ et al. 2015). However, data were not consistent between laboratories and the method was not considered useful in daily practice (Vergouw et al. 2014). In this work, we proposed a novel combined predictive model of embryo viability based on variables of morphokinetics of blastocysts and characteristic of EVs that achieved ROCAUC of 0.853 for Model-1 and Model-2. Statistically it was considered as 'good' model to select viable embryos. Model-1 included all variables evaluated, whereas Model-2 excluded BT without decreasing ROC-AUC value and the percent of correct predictions. The second model was useful from a practical point perspective, as it avoided disturbing embryo culture during blastulation. Certainly, incorporating EVs properties increases accuracy of embryo selection, as judged from comparisons of ROC-AUC values of good models versus Model-4 and Model-5. These data supported the possibility to improve embryo selection using conventional morphological criteria of the blastocyst combined to characteristics of EVs obtained by NTA. It was corroborated that NTA is very sensitive to analyze EVs population of individual embryos, it is easy and fast and thus can be performed very shortly after sample collection, allowing for appropriate and timely decisions about embryo fate. Besides, data are quantitative and easy to interpret.
Attempts to relate certain characteristics of spent culture media with bovine embryo viability have been published, but so far with non-convincing results, and the predictive value of proposed systems is still controversial and unfeasible (Reviewed by Rødgaard et al. 2015, Capalbo et al. 2016). That EVs contain molecules with regulatory potential emphasizes their biological importance, we analyzed the miRNA cargo of EVs from embryo culture media using small RNA sequencing, identifying 161 known miRNAs and 32 snoRNAs in EVs. Clustering analysis of miRNA/snoRNAs also allowed to discriminate viable embryos from both EB and LB groups. Moreover, differential miRNA expression patterns were observed between viable and non-viable embryos. However, when EB and LB were compared, no miRNAs were significantly differentially expressed despite embryo viability. In some of the compared miRNAs, there were $P$ values $<0.05$, but none of them met the FDR criterion set by us. Therefore these miRNAs were not considered for the analysis of their biological significance. Seemingly, blastulation (early or late) is the milestone after which there are no relevant changes in miRNA expression pattern in our experimental conditions. In addition, when BT was incorporated as a variable into the proposed model, it did not improve the percentage of positive prediction and thus it was excluded from the selected model (model-2).

We paid attention to miRNAs differentially expressed between viable and non-viable embryos. It has been proposed that miRNAs are selectively secreted into the culture medium by developing human embryos making them potential biomarkers of embryonic viability (Kropp et al. 2014, Rosenbluth et al. 2014, Kropp and Khatib 2015, Lin et al. 2019). In this work, 5 of the 12 upregulated miRNAs were identified solely in EVs secreted by viable embryos, of these, bta-miR-2284ab was upregulated the most. In the endometrium of goats, miR-2284ab is upregulated during the receptive phase (Song et al. 2015) as well as in cumulus cells of bovine oocytes that developed to blastocysts (Uhde et al. 2017).

Other miRNAS deregulated in our findings have been reported to participate in different reproductive events. The expression of bta-miR-1 is also higher in EVs from viable embryos. This miRNA acts in different functions including cell proliferation, cell cycle, apoptosis, cell migration and invasion (Li et al. 2012, Osaka et al. 2014). Members of miR-1 family regulate the balance between differentiation and proliferation by interacting with multiple targets in different cell types (Chen et al. 2006). In lung cancer and carcinoma cells, miR-1 suppresses cell proliferation by inducing arrest in the G0/G1 phase (Fujji et al. 2019). During embryo development, miR-1 induces angiogenesis in zebrafish (Lin et al. 2013), while in human and mouse miR-1 participates in the differentiation of skeletal and cardiac muscles (Chen et al. 2006). In the literature search we did not find any other report of miR-1 expressed in early embryos or in 
EVs secreted by preimplantation embryos. In this research we did not find a relation between embryo viability and the high expression of miR-1. Others reported that insulin-like growth factor 1 (IGF-1) is a target of miR-1 (Elia et al. 2009, Li et al. 2012, Hu et al. 2013). IGF-1 and 2 and their respective binding proteins (IGFBPs) are expressed in the endometrium of cattle (Wathes et al. 1998), but the concentration of IGF-1 decreases from day 0 to 18 in cattle carrying an embryo (Mense et al. 2018). IGF-1 concentration increases again between days 42 and 60 in pregnant cows suffering embryonic/ fetal mortality (Mense et al. 2018). The presence of miR-1 in EVs from viable embryos here could potentially lead to diminish the expression of IGF-1 in endometrium. Alternatively, viable embryos may take rid of unwanted miRNAs, like miR-1, to avoid cell arrest mediated by this or other miRNAs.

Recently we found bta-miR-1 to be downregulated in EVs secreted from day 3 to morula by embryos that developed to blastocysts, compared with those that becameblocked and did not develop further (unpublished data). It is tempting to propose that there would be a kinetic of release of miRNA potentially harmful for the embryo. It has been reported that levels of miRNA in cells do not necessarily correlate with the level in EVs derived from them; some miRNAs are abundant in EVs while rare in cells and conversely (Maida et al. 2016). In line with this, bta-miR-184 was found expressed only in EVs from viable embryos here. However, in human and mice embryos, miR-184 suppresses cell proliferation and induces apoptosis in trophoblastic cells; higher expression is related to embryo reabsorption (Zhang et al. 2019).

In this work, it was found bta-miR-10b upregulated in EVs from viable embryos. This is in contrast to Lin et al. (2019) who found that the expression of miR$10 \mathrm{~b}$ was higher in culture medium from slow and arrested preimplantation bovine embryos. However, this contradictory result might be due to different experimental designs; Lin et al. (2019) collected culture media from one-cell to blastocyst, while we collected media from morula to blastocyst and only from those embryos that reached blastocyst stage.

In our work bta-miR-200c among several other miRNAs were significantly downregulated in EVs from viable embryos. The expression of miR-200 family members is low in the receptive uterus in humans and mouse while is upregulated in infertile female (Liu et al. 2016, Zheng et al. 2017). miR-200c inhibits uterine epithelial cells receptivity and embryo implantation in mouse (Zheng et al. 2017). The low expression of miR200c in EVs from viable embryos in this work would favor endometrium receptivity. Interestingly, miR-200c is activated by the pluripotency transcription factors OCT4 and SOX2 to induce or maintain cell pluripotency
(Wang et al. 2013). In this sense, it would be a 'necessary' miRNA for a developing embryo and consequently would be conserved (i.e, not secreted). Previously, we found bta-miR-200c upregulated in more competent bovine embryos produced in vitro, compared to cloned embryos (Castro et al. 2010). Also, Kim et al. (2019) reported the upregulation of miR-200c-3p in mouse embryo outgrowths compared with non-outgrowth blastocysts. In line with this, here bta-miR-26 was found downregulated in $\mathrm{EV}$ s from viable embryos, while Castro et al. (2010) reported it was upregulated in more viable IVF embryos compared to clones.

Considering the facts discussed, it seems that EVs secretion plays a role in regulating the expression pattern of miRNAs in bovine blastocysts what is also related to embryo viability. Therefore, miRNA cargo of EVs could be evaluated in order to select more competent embryos. Regardless, validation analysis of the most prominent miRNAs is needed to include them in prediction models of embryo viability.

Exosomes are derived from major sorting hubs of endosomal trafficking; therefore, they are at the center of a signaling network that connects external stimuli with trophoblast cells intrinsic features. Differences in composition between EVs of viable and non-viable embryos may be due to the existence of regulated sorting mechanisms determining the phenotype and functional properties of these vesicles. Also, EV-associated miRNAs can travel long distances and become messages, regulating different biological process that may have an extraordinary impact on early embryo-maternal crosstalk (de Candia et al. 2016).

In conclusion, heterogeneous populations of EVs were secreted by individual embryos in an embryo viability-dependent manner. Furthermore, secreted miRNA and snoRNA were differently packaged into distinct populations of EVs related to embryo viability. Also, our binary logistic regression models (Model-1 and Model-2) effectively discriminated between viable and non-viable embryos, using blastocyst morphokinetics variables and EVs morphological parameters. It will be worth to define how miRNA cargo can collaborate by adding valuable predictive features to improve overall performance of pregnancy.

\section{Supplementary data}

This is linked to the online version of the paper at https://doi.org/10.1530/REP-19-0233.

\section{Declaration of interest}

The authors declare that there is no conflict of interest that could be perceived as prejudicing the impartiality of the research reported. 


\section{Funding}

This work was partially supported by FONDECYT 1170310 from the Ministry of Education of Chile. The funders had no role in the study design, data collection and analysis, decision to publish, or preparation of the manuscript.

\section{Author contribution statement}

E A M, L R-A: Conceptualization, E A M: Data curation, E A M, $M A B, L$ R-A: Formal analysis, L R-A: Funding acquisition, $E$ A M, L R-A: Investigation, E A M, A E V, L R-A: Methodology, L R-A: Project administration, L R-A, F O C: Resources, E A M, J C, M A B: Software, L R-A: Supervision, L R-A, F O C: Validation, E A M, L R-A: Visualization, E A M, L R-A: Writing (original draft preparation), E A M, L R-A, A E V, J C, M A B, F O C: Writing (review and editing) and all authors read, edited and approved the final manuscript.

\section{Acknowledgements}

The authors express their appreciation to Victor Gallardo Alvarez for his collaboration in retrieving ovaries from the abattoir. They are grateful to Dr John P Kastelic (Professor, Head Department of Production Animal Health, University of Calgary) for his contribution reviewing this manuscript.

\section{References}

Almiñana C, Corbin E, Tsikis G, Alcântara-Neto AS, Labas V, Reynaud K, Galio L, Uzbekov R, Garanina AS, Druart X, et al. 2017 Oviduct extracellular vesicles protein content and their role during oviductembryo cross-talk. Reproduction 154 153-168. (https://doi.org/10.1530/ REP-17-0054)

Battaglia R, Palini S, Vento ME, La Ferlita A, Lo Faro MJ, Caroppo E, Borzì P, Falzone L, Barbagallo D, Ragusa M, et al. 2019 Identification of extracellular vesicles and characterization of miRNA expression profiles in human blastocoel fluid. Scientific Reports 9 84. (https://doi. org/10.1038/s41598-018-36452-7)

Bó GA \& Mapletoft RJ 2013 Evaluation and classification of bovine embryos. Animal Reproduction 10 344-348.

Braicu C, Tomuleasa C, Monroig P, Cucuianu A, Berindan-Neagoe I \& Calin GA 2015 Exosomes as divine messengers: are they the Hermes of modern molecular oncology? Cell Death \& Differentiation 22 34-45. (https://doi.org/10.1038/cdd.2014.130)

Brandão DO, Maddox-Hyttel P, Løvendahl P, Rumpf R, Stringfellow D \& Callesen H 2004 Post hatching development: a novel system for extended in vitro culture of bovine embryos. Biology of Reproduction $\mathbf{7 1}$ 2048-2055. (https://doi.org/10.1095/biolreprod.103.025916)

Campbell A, Fishel S, Bowman N, Duffy S, Sedler M \& Fontes CF 2013 Modelling a risk classification of aneuploidy in human embryos using non-invasive morphokinetics. Reproductive Biomedicine Online 26 477-485. (https://doi.org/10.1016/j.rbmo.2013.02.006)

Capalbo A, Ubaldi FM, Cimadomo D, Noli L, Khalaf Y, Farcomeni A, Ilic D \& Rienzi L 2016 MiRNAs in spent blastocyst culture medium are derived from trophectoderm cells and can be explored for human embryo reproductive competence assessment. Fertility \& Sterility 105 225-235. e1-3. (https://doi.org/10.1016/j.fertnstert.2015.09.014)

Capece V, Garcia Vizcaino JC, Vidal R, Rahman RU, Pena Centeno T, Shomroni O, Suberviola I, Fischer A \& Bonn S 2015 Oasis: online analysis of small RNA deep sequencing data. Bioinformatics 312205 2207. (https://doi.org/10.1093/bioinformatics/btv113)

Castro FO, Sharbati S, Rodríguez-Alvarez LL, Cox JF, Hultschig C \& Einspanier R 2010 MicroRNA expression profiling of elongated cloned and in vitro-fertilized bovine embryos. Theriogenology 73 71-85. (https://doi.org/10.1016/j.theriogenology.2009.08.003)

Chen JF, Mandel EM, Thomson JM, Wu Q, Callis TE, Hammond SM, Conlon FL \& Wang DZ 2006 The role of microRNA-1 and microRNA-133 in skeletal muscle proliferation and differentiation. Nature Genetics 38 228-233. (https://doi.org/10.1038/ng1725)

Colombo M, Raposo G \& Théry C 2014 Biogenesis, secretion, and intercellular interactions of exosomes and other extracellular vesicles. Annual Review of Cell \& Developmental Biology 30 255-289. (https:// doi.org/10.1146/annurev-cellbio-101512-122326)

Cortezzi SS, Garcia JS, Ferreira CR, Braga DP, Figueira RC, laconelli A Jr, Souza GH, Borges E Jr \& Eberlin MN 2011 Secretome of the preimplantation human embryo by bottom-up label-free proteomics. Analytical \& Bioanalytical Chemistry 401 1331-1339. (https://doi. org/10.1007/s00216-011-5202-1)

de Candia P, De Rosa V, Casiraghi M \& Matarese G 2016 Extracellular RNAs: a secret arm of immune system regulation. Journal of Biological Chemistry 291 7221-7228. (https://doi.org/10.1074/jbc.R115.708842)

Dominguez F, Meseguer M, Aparicio-Ruiz B, Piqueras P, Quiñonero A \& Simón C 2015 New strategy for diagnosing embryo implantation potential by combining proteomics and time-lapse technologies. Fertility \& Sterility 104 908-914. (https://doi.org/10.1016/j.fertnstert.2015.06.032)

Dweep H, Sticht C, Pandey P \& Gretz N 2011 miRWalk--database: prediction of possible miRNA binding sites by "walking" the genes of three genomes. Journal of Biomedical Informatics 44 839-847. (https:// doi.org/10.1016/j.jbi.2011.05.002)

Elia L, Contu R, Quintavalle M, Varrone F, Chimenti C, Russo MA, Cimino V, De Marinis L, Frustaci A, Catalucci D, et al. 2009 Reciprocal regulation of microRNA-1 and insulin-like growth factor-1 signal transduction cascade in cardiac and skeletal muscle in physiological and pathological conditions. Circulation 120 2377-2385. (https://doi. org/10.1161/CIRCULATIONAHA.109.879429)

Ferreira YJ, Gardiner C, Poli M, Turner K, Child T \& Sargent IL 2013 O-253 human embryos release extracellular vesicles which may act as indicators of embryo quality. Session 66: embryo quality: does it predict pregnancy? Human Reproduction 28 1445-1736. (https://doi. org/10.1093/humrep/det200)

Fischer-Brown AE, Lindsey BR, Ireland FA, Northey DL, Monson RL, Clark SG, Wheeler MB, Kesler DJ, Lane SJ, Weigel KA, et al. 2004 Embryonic disc development and subsequent viability of cattle embryos following culture in two media under two oxygen concentrations. Reproduction, Fertility, and Development 16 787-793. (https://doi. org/10.1071/RD04026)

Fujii R, Osaka E, Sato K \& Tokuhashi Y 2019 MiR-1 suppresses proliferation of osteosarcoma cells by up-regulating p21 via PAX3. Cancer Genomics \& Proteomics 16 71-79. (https://doi.org/10.21873/cgp.20113)

Graf A, Krebs S, Zakhartchenko V, Schwalb B, Blum H \& Wolf E 2014 Fine mapping of genome activation in bovine embryos by RNA sequencing. PNAS 111 4139-4144. (https://doi.org/10.1073/pnas.1321569111)

Gutiérrez-Adán A, Rizos D, Fair T, Moreira PN, Pintado B, de la Fuente J, Boland MP \& Lonergan P 2004 Effect of speed of development on mRNA expression pattern in early bovine embryos cultured in vivo or in vitro. Molecular Reproduction \& Development 68 441-448. (https://doi. org/10.1002/mrd.20113)

Hoelker M, Schmoll F, Schneider H, Rings F, Gilles M, Tesfaye D, Jennen D, Tholen E, Griese J \& Schellander K 2006 Bovine blastocyst diameter as a morphological tool to predict embryo cell counts, embryo sex, hatching ability and developmental characteristics after transfer to recipients. Reproduction, Fertility, and Development 18 551-557. (https://doi. org/10.1071/RD05149)

Hu W, Meng X, Lu T, Wu L, Li T, Li M \& Tian Y 2013 MicroRNA-1 inhibits the proliferation of Chinese sika deer-derived cartilage cells by binding to the 3'-untranslated region of IGF-1. Molecular Medicine Reports 8 523-528. (https://doi.org/10.3892/mmr.2013.1515)

Kaith S, Saini M, Raja AK, Sahare AA, Jyotsana B, Madheshiya P, Palta P, Chauhan MS, Manik RS \& Singla SK 2015 Early cleavage of handmade cloned buffalo (Bubalus bubalis) embryos is an indicator of their developmental competence and quality. Reproduction in Domestic Animals = Zuchthygiene 50 214-220. (https://doi.org/10.1111/ rda.12472)

Katz-Jaffe MG \& Gardner DK 2008 Symposium: innovative techniques in human embryo viability assessment. Can proteomics help to shape the 
future of human assisted conception? Reproductive Biomedicine Online 17 497-501. (https://doi.org/10.1016/s1472-6483(10)60235-0)

Killian GJ 2004 Evidence for the role of oviduct secretions in sperm function, fertilization and embryo development. Animal Reproduction Science 82-83 141-153. (https://doi.org/10.1016/j.anireprosci.2004.04.028)

Kim J, Lee J \& Jun JH 2019 Identification of differentially expressed microRNAs in outgrowth embryos compared with blastocysts and nonoutgrowth embryos in mice. Reproduction, Fertility, and Development 31 645-657. (https://doi.org/10.1071/RD18161)

Kowal J, Tkach M \& Théry C 2014 Biogenesis and secretion of exosomes. Current Opinion in Cell Biology 29 116-125. (https://doi.org/10.1016/j. ceb.2014.05.004)

Kropp J \& Khatib H 2015 Characterization of microRNA in bovine in vitro culture media associated with embryo quality and development. Journal of Dairy Science 98 6552-6563. (https://doi.org/10.3168/jds.20159510)

Kropp J, Salih SM \& Khatib H 2014 Expression of microRNAs in bovine and human pre-implantation embryo culture media. Frontiers in Genetics $\mathbf{5}$ 91. (https://doi.org/10.3389/fgene.2014.00091)

Kurian NK \& Modi D 2019 Extracellular vesicle mediated embryoendometrial cross talk during implantation and in pregnancy. Journal of Assisted Reproduction \& Genetics 36 189-198. (https://doi.org/10.1007/ s10815-018-1343-x)

Li Y, Shelat H \& Geng YJ 2012 IGF-1 prevents oxidative stress inducedapoptosis in induced pluripotent stem cells which is mediated by microRNA-1. Biochemical \& Biophysical Research Communications 426 615-619. (https://doi.org/10.1016/j.bbrc.2012.08.139)

Li X, Xu Y, Fu J, Zhang WB, Liu SY \& Sun XX 2015 Non-invasive metabolomic profiling of embryo culture media and morphology grading to predict implantation outcome in frozen-thawed embryo transfer cycles. Journal of Assisted Reproduction \& Genetics 32 15971605. (https://doi.org/10.1007/s10815-015-0578-z)

Lin CY, Lee HC, Fu CY, Ding YY, Chen JS, Lee MH, Huang WJ \& Tsai HJ 2013 MiR-1 and miR-206 target different genes to have opposing roles during angiogenesis in zebrafish embryos. Nature Communications 4 2829. (https://doi.org/10.1038/ncomms3829)

Lin X, Pavani KC, Smits K, Deforce D, Heindryckx B, Van Soom A \& Peelman L 2019 Bta-miR-10b Secreted by Bovine Embryos Negatively Impacts Preimplantation Embryo Quality. Frontiers Genetics 22757. (https://doi.org/ 10.3389/fgene.2019.00757)

Liu W, Niu Z, Li Q, Pang RT, Chiu PC \& Yeung WS 2016 MicroRNA and embryo implantation. American Journal of Reproductive Immunology 75 263-271. (https://doi.org/10.1111/aji.12470)

Lopera-Vasquez R, Hamdi M, Fernandez-Fuertes B, Maillo V, BeltránBreña $P$, Calle A, Redruello A, López-Martín S, Gutierrez-Adán A, Yañez-Mó M, et al. 2016 Extracellular vesicles from BOEC in in vitro embryo development and quality. PLOS ONE 11 e0148083. (https://doi. org/10.1371/journal.pone.0148083)

Lopera-Vasquez R, Hamdi M, Maillo V, Gutierrez-Adan A, BermejoAlvarez P, Ramírez MÁ, Yáñez-Mó M \& Rizos D 2017 Effect of bovine oviductal extracellular vesicles on embryo development and quality in vitro. Reproduction 153 461-470. (https://doi.org/10.1530/REP-160384)

Lopera-Vasquez R, Hamdi M, Maillo V, Gutierrez-Adan A, BermejoAlvarez P, Ramírez MÁ, Yáñez-Mó M \& Rizos D 2018 Effect of bovine oviductal extracellular vesicles on embryo development and quality in vitro. Reproduction 153 461-470. (https://doi.org/ 10.1530/REP-160384)

Love MI, Huber W \& Anders S 2014 Moderated estimation of fold change and dispersion for RNA-seq data with DESeq2. Genome Biology 15550. (https://doi.org/10.1186/s13059-014-0550-8)

Lv C, Yu WX, Wang Y, Yi DJ, Zeng MH \& Xiao HM 2018 MiR-21 in extracellular vesicles contributes to the growth of fertilized eggs and embryo development in mice. Bioscience Reports 38 BSR20180036. (https://doi.org/10.1042/BSR20180036)

Madan P, Calder MD \& Watson AJ 2005 Mitogen-activated protein kinase (MAPK) blockade of bovine preimplantation embryogenesis requires inhibition of both $\mathrm{p} 38$ and extracellular signal-regulated kinase (ERK) pathways. Reproduction 130 41-51. (https://doi.org/10.1530/ rep.1.00554)

Maida Y, Takakura M, Nishiuchi T, Yoshimoto T \& Kyo S 2016 Exosomal transfer of functional small RNAs mediates cancer-stroma communication in human endometrium. Cancer Medicine 5 304-314. (https://doi.org/10.1002/cam4.545)

Maillo V, Sánchez-Calabuig MJ, Lopera-Vasquez R, Hamdi M, GutierrezAdan A, Lonergan P \& Rizos D 2016 Oviductal response to gametes and early embryos in mammals. Reproduction 152 R127-R141. (https://doi. org/10.1530/REP-16-0120)

Maître JL 2017 Mechanics of blastocyst morphogenesis. Biology of the Cell 109 323-338 (https://doi.org/10.1111/boc.201700029)

Mellisho EA, Velásquez AE, Nuñez MJ, Cabezas JG, Cueto JA, Fader C, Castro FO \& Rodríguez-Álvarez L 2017 Identification and characteristics of extracellular vesicles from bovine blastocysts produced in vitro. PLOS ONE 12 e0178306. (https://doi.org/10.1371/journal.pone.0178306)

Ménézo Y, Lichtblau I \& Elder K 2013 New insights into human preimplantation metabolism in vivo and in vitro. Journal of Assisted Reproduction \& Genetics 30 293-303. (https://doi.org/10.1007/s10815013-9953-9)

Mense K, Heidekorn-Dettmer J, Wirthgen E, Brockelmann Y, Bortfeldt R, Peter S, Jung M, Höflich C, Hoeflich A \& Schmicke M 2018 Increased concentrations of insulin-like growth factor binding protein (IGFBP)-2, IGFBP-3, and IGFBP-4 are associated with fetal mortality in pregnant cows. Frontiers in Endocrinology 9 310. (https://doi.org/10.3389/ fendo.2018.00310)

Meseguer M, Herrero J, Tejera A, Hilligsøe KM, Ramsing NB \& Remohí J 2011 The use of morphokinetics as a predictor of embryo implantation. Human Reproduction 26 2658-2671. (https://doi.org/10.1093/humrep/ der256)

Mi H, Muruganujan A, Casagrande JT \& Thomas PD 2013 Large-scale gene function analysis with the PANTHER classification system. Nature Protocols 8 1551-1566. (https://doi.org/10.1038/nprot.2013.092)

Milazzotto MP, Goissis MD, Chitwood JL, Annes K, Soares CA, Ispada J, Assumpção ME \& Ross PJ 2016 Early cleavages influence the molecular and the metabolic pattern of individually cultured bovine blastocysts. Molecular Reproduction \& Development 83 324-336. (https://doi. org/10.1002/mrd.22619)

Milewski R, Kuć P, Kuczyńska A, Stankiewicz B, Łukaszuk K \& Kuczyński W 2015 A predictive model for blastocyst formation based on morphokinetic parameters in time-lapse monitoring of embryo development. Journal of Assisted Reproduction and Genetics 32571 579. (https://doi.org/10.1007/s10815-015-0440-3)

Milewski R, Milewska AJ, Kuczyńska A, Stankiewicz B \& Kuczyński W 2016 Do morphokinetic data sets inform pregnancy potential? Journal of Assisted Reproduction \& Genetics 33 357-365. (https://doi.org/10.1007/ s10815-016-0649-9)

Motato Y, de los Santos MJ, Escriba MJ, Ruiz BA, Remohí J \& Meseguer M 2016 Morphokinetic analysis and embryonic prediction for blastocyst formation through an integrated time-lapse system. Fertility \& Sterility 105 376-84.e9. (https://doi.org/10.1016/j.fertnstert.2015.11.001)

Muthukumar K, Kamath MS, Mangalaraj AM, Aleyamma T, Chandy A \&, George K 2013 Comparison of clinical outcomes following vitrified warmed day 5/6 blastocyst transfers using solid surface methodology with fresh blastocyst transfers. Journal of Human Reproductive Sciences 6 59-64. (https://doi.org/10.4103/0974-1208.112384)

O'Neill C 2008 The potential roles for embryotrophic ligands in preimplantation embryo development. Human Reproduction Update 14 275-288. (https://doi.org/10.1093/humupd/dmn002)

Osaka E, Yang X, Shen JK, Yang P, Feng Y, Mankin HJ, Hornicek FJ \& Duan Z 2014 MicroRNA-1 (miR-1) inhibits chordoma cell migration and invasion by targeting slug. Journal of Orthopaedic Research 32 10751082. (https://doi.org/10.1002/jor.22632)

Penkova TG 2017 Principal component analysis and cluster analysis for evaluating the natural and anthropogenic territory safety. Procedia Computer Science 112 99-108. (https://doi.org/10.1016/j. procs.2017.08.179)

Qu P, Qing S, Liu R, Qin H, Wang W, Qiao F, Ge H, Liu J, Zhang Y, Cui W, et al. 2017 Effects of embryo-derived exosomes on the development of bovine cloned embryos. PLOS ONE 12 e0174535. (https://doi. org/10.1371/journal.pone.0174535)

Raposo G \& Stoorvogel W 2013 Extracellular vesicles: exosomes, microvesicles, and friends. Journal of Cell Biology 200 373-383. (https:// doi.org/10.1083/jcb.201211138)

Rekik W, Dufort I \& Sirard MA 2011 Analysis of the gene expression pattern of bovine blastocysts at three stages of development. Molecular 
Reproduction \& Development 78 226-240. (https://doi.org/10.1002/ mrd.21286)

Rødgaard T, Heegaard PM \& Callesen H 2015 Non-invasive assessment of in-vitro embryo quality to improve transfer success. Reproductive Biomedicine Online 31 585-592. (https://doi.org/10.1016/j. rbmo.2015.08.003)

Rodríguez L, Navarrete Fl, Tovar H, Cox JF \& Castro FO 2008 High developmental potential in vitro and in vivo of cattle embryos cloned without micromanipulators. Journal of Assisted Reproduction \& Genetics 25 13-16. (https://doi.org/10.1007/s10815-007-9194-x)

Rosenbluth EM, Shelton DN, Wells LM, Sparks AET \& Van Voorhis BJ 2014 Human embryos secrete microRNAs into culture media-A potential biomarker for implantation. Fertility \& Sterility 101 1493-1500. (https:// doi.org/10.1016/j.fertnstert.2014.01.058)

Rossant J \& Tam PP 2009 Blastocyst lineage formation, early embryonic asymmetries and axis patterning in the mouse. Development $136701-$ 713. (https://doi.org/10.1242/dev.017178)

Saadeldin IM, Kim SJ, Choi YB \& Lee BC 2014 Improvement of cloned embryos development by co-culturing with parthenotes: a possible role of exosomes/microvesicles for embryos paracrine communication. Cell Reprogram 16 223-234. (https://doi.org/10.1089/cell.2014.0003)

Seli E, Sakkas D, Scott R, Kwok SC, Rosendahl SM \& Burns DH 2007 Non-invasive metabolomic profiling of human embryo culture media using Raman and near infrared spectroscopy correlates with reproductive potential of embryos in women undergoing in vitro fertilization. Fertility \& Sterility 88 1350-1357. (https://doi.org/10.1016/j. fertnstert.2007.07.1390)

Seli E, Bruce C, Botros L, Henson M, Roos P, Judge K, Hardarson T, Ahlström A, Harrison P, Henman M, et al. 2011 Receiver operating characteristic (ROC) analysis of day 5 morphology grading and metabolomic Viability Score on predicting implantation outcome. Journal of Assisted Reproduction \& Genetics 28 137-144. (https://doi. org/10.1007/s10815-010-9501-9)

Shapiro BS, Daneshmand ST, Garner FC, Aguirre M \& Thomas S 2008 Large blastocyst diameter, early blastulation, and low preovulatory serum progesterone are dominant predictors of clinical pregnancy in fresh autologous cycles. Fertility \& Sterility 90 302-309. (https://doi. org/10.1016/j.fertnstert.2007.06.062)

Sharma S, Gillespie BM, Palanisamy V \& Gimzewski JK 2011 Quantitative nanostructural and single-molecule force spectroscopy biomolecular analysis of human-saliva-derived exosomes. Langmuir 27 14394-14400. (https://doi.org/10.1021/la2038763)

Skog J, Würdinger T, van Rijn S, Meijer DH, Gainche L, Sena-Esteves M, Curry WT Jr, Carter BS, Krichevsky AM \& Breakefield XO 2008 Glioblastoma microvesicles transport RNA and proteins that promote tumour growth and provide diagnostic biomarkers. Nature Cell Biology 10 1470-1476. (https://doi.org/10.1038/ncb1800)

Song Y, An X, Zhang L, Fu M, Peng J, Han P, Hou J, Zhou Z \& Cao B 2015 Identification and profiling of microRNAs in goat endometrium during embryo implantation. PLOS ONE 10 e0122202. (https://doi. org/10.1371/journal.pone.0122202)

Stringfellow DA \& Givens MD 2010 Manual of the International Embryo Transfer Society (IETS), 4th ed. Champaign, IL: IETS.

Swain JE 2013 Could time-lapse embryo imaging reduce the need for biopsy and PGS? Journal of Assisted Reproduction \& Genetics 301081 1090. (https://doi.org/10.1007/s10815-013-0048-4)

Tkach M \& Thery C 2016 Communication by extracellular vesicles: where we are and where we need to go. Cell 164 1226-1232. (https://doi. org/10.1016/j.cell.2016.01.043)

Uhde K, van Tol HTA, Stout TAE \& Roelen BAJ 2017 MicroRNA expression in bovine cumulus cells in relation to oocyte quality. Non-Coding RNA 3 12. (https://doi.org/10.3390/ncrna3010012)

Utsunomiya T, Ito H, Nagaki M \& Sato J 2004 A prospective, randomized study: day 3 versus hatching blastocyst stage. Human Reproduction 19 1598-1603. (https://doi.org/10.1093/humrep/deh288) van der Pol E, Böing AN, Harrison P, Sturk A \& Nieuwland R 2012 Classification, functions, and clinical relevance of extracellular vesicles. Pharmacological Reviews 64 676-705. (https://doi.org/10.1124/ pr.112.005983)

Velasquez AE, Castro FO, Veraguas D, Cox JF, Lara E, Briones M \& Rodriguez-Alvarez L 2016 Splitting of IVP bovine blastocyst affects morphology and gene expression of resulting demi-embryos during in vitro culture and in vivo elongation. Zygote 24 18-30. (https://doi. org/10.1017/S0967199414000677)

Velásquez AE, Manríquez J, Castro FO, Cox JF \& Rodriguez-Alvarez L 2017 Embryo splitting affects the transcriptome during elongation stage of in vitro-produced bovine blastocysts. Theriogenology 87 124-134. (https://doi.org/10.1016/j.theriogenology.2016.08.014)

Vergouw CG, Heymans MW, Hardarson T, Sfontouris IA, Economou KA, Ahlström A, Rogberg L, Lainas TG, Sakkas D, Kieslinger DC, et al. 2014 No evidence that embryo selection by near-infrared spectroscopy in addition to morphology is able to improve live birth rates: results from an individual patient data meta-analysis. Human Reproduction 29 455461. (https://doi.org/10.1093/humrep/det456)

Wang G, Guo X, Hong W, Liu Q, Wei T, Lu C, Gao L, Ye D, Zhou Y, Chen J et al. 2013 Critical regulation of miR-200/ZEB2 pathway in Oct4/Sox2-induced mesenchymal-to-epithelial transition and induced pluripotent stem cell generation. PNAS 110 2858-2863 (https://doi.org/ 10.1073/pnas.1212769110)

Wang X, Tian F, Chen C, Feng Y, Sheng X, Guo Y \& Ni H 2019 Exosomederived uterine microRNAs isolated from cows with endometritis impede blastocyst development. Reproductive Biology 19 204-209. (https://doi. org/10.1016/j.repbio.2019.06.003)

Wathes DC, Reynolds TS, Robinson RS \& Stevenson KR 1998 Role of the insulin-like growth factor system in uterine function and placental development in ruminants. Journal of Dairy Science 81 1778-1789. (https://doi.org/10.3168/jds.S0022-0302(98)75747-9)

Watson AJ \& Barcroft LC 2001 Regulation of blastocyst formation. Frontiers in Bioscience 6 D708-D730. (https://doi.org/10.2741/watson)

Watson AJ, Westhusin ME, De Sousa PA, Betts DH \& Barcroft LC 1999 Gene expression regulating blastocyst formation. Theriogenology $\mathbf{5 1}$ 117-133. (https://doi.org/10.1016/S0093-691X(98)00236-2)

Wong CC, Loewke KE, Bossert NL, Behr B, De Jonge CJ, Baer TM \& Reijo Pera RA 2010 Non-invasive imaging of human embryos before embryonic genome activation predicts development to the blastocyst stage. Nature Biotechnology 28 1115-1121. (https://doi.org/10.1038/ nbt.1686)

Zhang Y, Zhou J, Li MQ, Xu J, Zhang JP \& Jin LP 2019 MicroRNA-184 promotes apoptosis of trophoblast cells via targeting WIG1 and induces early spontaneous abortion. Cell Death \& Disease 10 223. (https://doi. org/10.1038/s41419-019-1443-2)

Zheng Q, Zhang D, Yang YU, Cui X, Sun J, Liang C, Qin H, Yang X, Liu S \& Yan Q 2017 MicroRNA-200c impairs uterine receptivity formation by targeting FUT4 and $\alpha 1,3$-fucosylation. Cell Death \& Differentiation 24 2161-2172. (https://doi.org/10.1038/cdd.2017.136)

Zumoffen CM, Gil R, Caille AM, Morente C, Munuce MJ \& Ghersevich SA 2013 A protein isolated from human oviductal tissue in vitro secretion, identified as human lactoferrin, interacts with spermatozoa and oocytes and modulates gamete interaction. Human Reproduction 28 12971308. (https://doi.org/10.1093/humrep/det016)

Received 27 May 2019

First decision 6 August 2019

Revised manuscript received 27 August 2019

Accepted 1 October 2019 